\title{
Characterization of B-BOX gene family and their expression profiles under hormonal, abiotic and metal stresses in Poaceae plants

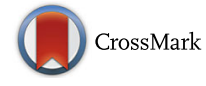

\author{
Abdullah Shalmani ${ }^{\dagger}$, Xiu-Qing Jing ${ }^{\dagger}$, Yi Shi, Izhar Muhammad, Meng-Ru Zhou, Xiao-Yong Wei, Qiong-Qiong Chen, \\ Wen-Qiang Li, Wen-Ting Liu and Kun-Ming Chen ${ }^{*}$
}

\begin{abstract}
Background: B-box (BBX) proteins play important roles in plant growth regulation and development including photomorphogenesis, photoperiodic regulation of flowering, and responses to biotic and abiotic stresses.

Results: In the present study we retrieved total 131 BBX members from five Poaceae species including 36 from maize, 30 from rice, 24 from sorghum, 22 from stiff brome, and 19 from Millet. All the BBX genes were grouped into five subfamilies on the basis of their phylogenetic relationships and structural features. The expression profiles of 12 OsBBX genes in different tissues were evaluated through qRT-PCR, and we found that most rice $B B X$ members showed high expression level in the heading stage compared to seedling and booting stages. The expression of OsBBX1, OsBBX2, OsBBX8, OsBBX19, and OsBBX24 was strongly induced by abiotic stresses such as drought, cold and salt stresses. Furthermore, the expression of OsBBX2, OsBBX7, OsBBX17, OsBBX19, and OsBBX24 genes was up-regulated under GA, SA and MeJA hormones at different time points. Similarly, the transcripts level of OsBBX1, OsBBX7, OsBBX8, OsBBX17, and OsBBX19 genes were significantly affected by heavy metals such as $\mathrm{Fe}, \mathrm{Ni}, \mathrm{Cr}$ and $\mathrm{Cd}$.
\end{abstract}

Conclusion: Change in the expression pattern of BBX members in response to abiotic, hormone and heavy metal stresses signifies their potential roles in plant growth and development and in response to multivariate stresses. The findings suggest that $B B X$ genes could be used as potential genetic markers for the plants, particularly in functional analysis and determining their roles under multivariate stresses.

Keywords: BBX, Poaceae, synteny, expression analysis

\section{Background}

Zinc finger transcription factors (TFs) are one of the most important families in plants. They regulate different plant growth and development processes. Zinc finger TFs are classified into several subfamilies based on the structural and functional features of their individual members. Among them, B-box proteins (BBXs) drew more attention in recent years due to their multiple functions. The BBXs contain one or two conserved $\mathrm{B}$-box domains near to $\mathrm{N}$-terminus and some have an additional CCT (CONSTANS, CO-like, and TIMING Of

\footnotetext{
*Correspondence: kunmingchen@nwsuaf.edu.cn; kunmingchen@163.com.cn ${ }^{\dagger}$ Abdullah Shalmani and Xiu-Qing Jing contributed equally to this work. State Key Laboratory of Crop Stress Biology in Arid Areas, College of Life Sciences, Northwest A\&F University, Yangling 712100, China
}

CAB1) conserved domain near to C-terminal. The B-box domains are divided into two classes, known as B-box1 (B1) and B-box2 (B2). Two B-box conserved domains are recognized on their consensus sequence and the distance between the zinc-binding residues [1]. The segmental duplication and deletion events during evolution result in the differences of the consensus sequences in the two B-box domains [2,3]. The highly conserved CCT domain is comprised of $42-43$ amino acids and is important for the regulation of functional transcription and nuclear protein transport [4].

Recent genome-wide expression studies suggested that the $\mathrm{BBX}$ proteins are involved in plant hormone signaling responses. Abscisic acid (ABA) is a phytohormone which is activated when the plants are exposed to

(c) The Author(s). 2019 Open Access This article is distributed under the terms of the Creative Commons Attribution 4.0 International License (http://creativecommons.org/licenses/by/4.0/), which permits unrestricted use, distribution, and 
different stresses [5]. Microarray analysis detected that the expression pattern of $B B X$ genes is different in response to ABA [6, 7]. The microarray study also found that the expression of $B B X 32$ was up-regulated by the cyclopentenone precursor of JA, 12-oxo-pentadienoic acid (OPDA), but not by JA or MeJA in Arabidopsis plants [8]. In addition, it was found that BZS1/BBX20 integrates signals from brassinosteroids (BR) and light pathways [9]. BRASSINAZOLE RESISTANT 1 (BZR1) is a transcription factor that triggered hypocotyl growth by directly binding to BBX20 [10]. Interestingly, GATA2, a GATA-binding zinc-finger protein stopped hypocotyl growth by reducing BR signaling action [11]. So, it can be postulated that $B B X 20$ works together with GATA2 in facilitating light and BR crosstalk. Recently, it was reported that $B B X 18$ play a potential role in the gibberellin (GA) signaling pathway [12]. Molecular and phenotypic studies proved that BBX18 enhances the hypocotyl growth by up-regulation of bioactive GA levels. Certainly, $B B X 18$ promotes the activities of GA3ox1 and GA20ox1 metabolic genes but decreased the activities of GA2ox1 and GA2ox8 catabolic genes under light [12]. The involvement of $B B X$ genes in the COP/HY5 signaling pathway indicates that $B B X 18$ may work as an integrator of both GA and COP1/HY5 pathways [13]. In addition, the microarray database showed that the transcript level of $11 B B X$ genes was distinct in rice when the plants were exposed to auxin, GA, and cytokinin treatments, and the studied rice $B B X s$ have hormone-responsive cis-acting elements in their promoters [14]. These results indicate the probable involvement of BBX proteins in hormones signaling in plants. However, the functional mechanisms of BBXs in hormonal signaling pathways are still little known.

BBXs might also play vital roles in abiotic stress tolerance of plants. The salt tolerance protein (STO, AtBBX24) enhances the growth of root under a high salinity condition in Arabidopsis [15], and was also triggered by the salt tolerance activities in yeast cells [16]. STO inoculates with CLONE EIGHTY-ONE/RADICAL-INDUCED CELL DEATH1 (CEO/RCD1) [17], which negatively regulates a wide range of stress-related genes [18]. Another $B B X$ gene, $A t B B X 18$, acts as a negative regulator both in photomorphogenesis and thermotolerance in Arabidopsis [12]. Furthermore, AtBBX18 negatively regulates the expression of heat-responsive genes such as DGD1, Hsp70, Hsp101, and APX2, thereby reducing germination and seedling survival after the heat treatment [12]. In Chrysanthemum, CmBBX24 performed a dual function, delaying flowering and also increase cold or drought tolerance [19]. Moreover, the overexpression of AtBBX24 enhances salt tolerance compared to wild-type plants, and a significant increase in root length in Arabidopsis [15]. Twenty-nine out of
30 rice $B B X$ genes possess at least one stress-responsive cis-elements such as ARE, Wbox, GC-motif, Box-W1, HSE, and MBS, signifying that these genes may express during biotic and abiotic stresses [14].

The studies on B-box proteins have emerging role in the plant development and of great interest for various researchers nowadays. Although, the $B B X$ gene family and their expression patterns under a few hormones were previously reported in rice [14], the evolutionary relationships of BBXs especially in Poaceae not yet been clearly understood. Additionally, the roles of $B B X$ genes in evolutionary origin and structural changes, the plant stress response and functional diversity of these proteins are still little understood in land plants. Therefore, in the present study, the $B B X$ gene family members in five Poaceae species and their expression profiles under various hormones, abiotic and heavy metal stresses in rice were systematically investigated. The obtained results will enlighten the novel insights into their action and the evolutionary significance of their functional divergence. Furthermore, the gene expression pattern will assist to improve the potential $B B X$ candidate genes involved in plant development regulation and multivariate stress responses.

\section{Materials \\ Identification of $B B X$ gene family member}

The Arabidopsis $B B X$ gene family has already been reported [1]. All the downloaded BBX protein sequences from Arabidopsis Information Resource (TAIR) database (http://www.arabidopsis.org) were used as queries for BLASTP search with default parameters against maize genome database (https://maizegdb.org), the rice genome database (Rice Annotation Project (RAP) v1.0, http:// rapdb.dna.affrc.go.jp/) and plant genome database (http:// plantgdb.org/SbGDB/SiGDB/BdGDB/). Afterward, all the protein sequences were further scanned to check their completeness and presence of the target domain via the following online tools: SMART (http://smart.embl-heidelberg.de/) [20], Inter Pro Scan program (http://www.ebi.ac.uk/interpro/), Conserved Domain Database (CDD) (http://www.ncbi.nlm.nih.gov/cdd/), and Scan Prosite (http://prosite.expasy.org/scanprosite/). The chemical features of BBX proteins such as isoelectric point $(\mathrm{PI})$, molecular weight $(\mathrm{kD})$, instability index, aliphatic index, grand average of hydropathy (GRAVY) and major amino acids of each BBX proteins were investigated using the ExPASy proteomics server (http:// web.expasy.org/protparam/)

\section{Chromosomal localization, Exon and Intron Distribution and Conserved Motif Analysis}

The corresponding genome database was used to obtain the candidate $B B X$ gene annotations and their chromosomal 
locations. The exact locations of genes on chromosomes were identified by using MapDraw. The conserved and shared domains for all BBX protein sequences were created by online version 4.9.1 of the Multiple Expectation for Motif Elicitation (MEME) tool (http://meme-suite.org/) [21, 22]. Gene Structure Display Server (http://gsds.cbi.pku.edu.cn) was used to construct the exon-intron structure consisting exon positions and gene length of $B B X$ genes.

\section{Sequence alignment and Phylogenetic analysis}

Multiple alignments of BBX protein sequences were performed with DNAMAN software (Version 5.2.2, LynnonBiosoft, Canada), and the sequence logos were constructed through online Weblogo platform (http:// weblogo.berkeley.edu/logo.cgi). The candidate BBX proteins were initially multiply aligned by using the ClustalW v2.0 online tool (http://www.ebi.ac.uk/Tools/webservices/ services/msa/clustalw2_soap) to further search the evolutionary relationships of the $B B X$ gene family and then the maximum likelihood phylogenetic tree was constructed by using the MEGA 6.06 software package with default parameters and the reliability of interior branches was assessed with 1000 bootstrap repetitions.

\section{Tandem Duplication and Synteny Analysis}

The Plant Genome Duplication Database (http://chibba.agtec.uga.edu/duplication/) was used to obtain syntenic blocks. Then circos version software (http://circos.ca/) was used to draw the diagrams. The physical location of a gene on the chromosome was used to find out the Tandem duplication of $B B X$ gene. Genes having an adjacent homologous $B B X$ gene on the same chromosome with no more than one intervening gene were considered to be tandemly duplicated.

\section{Plant Material and Growth Conditions}

The experimental work was performed in the field of State Key Laboratory of Crops Stress Biology for Arid Areas (Northwest A\&F University, Yangling, China). First of all, the seeds were sterilized with $0.5 \%(\mathrm{w} / \mathrm{v})$ sodium hypochlorite $(\mathrm{NaClO})$ for $4 \mathrm{~h}$, then washed thrice with distilled water. Seeds were then soaked in water for $48 \mathrm{~h}$ in darkness. Subsequently, the seeds were propagated on humid cheesecloth at $28{ }^{\circ} \mathrm{C}$ for $72 \mathrm{~h}$ and wetted with deionized water each day. Healthy and uniform seedlings were selected and grown in hydroponic solution prepared in Milli- $\mathrm{Q}$ water [23], containing $16 \mathrm{mM}$ $\mathrm{KNO}_{3}, 6 \mathrm{mM} \mathrm{Ca}\left(\mathrm{NO}_{3}\right)_{2} \cdot 4 \mathrm{H}_{2} \mathrm{O}, 4 \mathrm{mM} \mathrm{NH} \mathrm{NH}_{4} \mathrm{H}_{2} \mathrm{PO}_{4}, 2$ $\mathrm{mM} \mathrm{MgSO}_{4} \cdot 7 \mathrm{H}_{2} \mathrm{O}, 50 \mu \mathrm{M} \mathrm{KCl}, 25 \mu \mathrm{M} \mathrm{H} \mathrm{H}_{3} \mathrm{BO}_{3}, 25 \mu \mathrm{M}$ Fe-EDTA, $2 \mu \mathrm{M} \quad \mathrm{MnSO}_{4} \cdot 4 \mathrm{H}_{2} \mathrm{O}, 2 \mu \mathrm{M} \quad \mathrm{ZnSO}_{4}, 0.5 \mu \mathrm{M}$ $\mathrm{Na}_{2} \mathrm{MoO}_{4} \cdot 2 \mathrm{H}_{2} \mathrm{O}$, and $0.5 \mu \mathrm{M} \mathrm{CuSO}_{4} \cdot 5 \mathrm{H}_{2} \mathrm{O}$. The plants were floated in nutrient solution fixed with foam plugged in vessels (one plant in the single vessel). The nutrient solutions were continuously aerated and the environment was firmly controlled in growth chamber condition at $(16 \mathrm{~h} / 8 \mathrm{~h}$ day/night, temperature cycle of $30^{\circ} \mathrm{C} / 25^{\circ} \mathrm{C}, 800 \mu \mathrm{mol} \mathrm{m}{ }^{-2} \mathrm{~s}^{-1}$ light intensity and $60-$ $65 \%$ relative humidity level). The solution was changed after $24 \mathrm{~h}$ duration and the $\mathrm{pH}$ was adjusted to 5.8 by using $\mathrm{NaOH}$ or $\mathrm{HCl}$.

\section{Stress Treatments and Sample Collection}

To identify the transcript profiles of $B B X$ genes in rice, the young seedling (two-week-old) were exposed to various abiotic stresses, phytohormones and heavy metals. For heat stress treatment, the seedlings at four-leaf stage were subjected at $40^{\circ} \mathrm{C}$ temperature with $60 \%$ humidity, $16 \mathrm{~h}$ photoperiod in the growth chamber under fluorescent light for $24 \mathrm{~h}$. For cold stress, at the same stage seedlings were transferred into the cold cabinet (SANYO) under a 14-h light: 10-h dark, with light conditions of $300 \mu \mathrm{mol}$ photons $\mathrm{m}^{-2} \mathrm{~s}^{-1}$. For dehydration $20 \%$ polyethylene glycol (PEG-6000), the solution was purified by passing it through an ion exchange column to remove any impurities and was filtered using Miracloth (22-25 $\mu \mathrm{m}$, Thomas Scientific, Swedesboro, NJ, USA). Salt ( $200 \mathrm{mM} \mathrm{NaCl}$ ) was prepared from stock solution by dissolving in water. Then seedlings were submerged in $20 \%$ PEG6000 or $200 \mathrm{mM} \mathrm{NaCl}$ solutions for drought and salt treatments respectively. The final hormonal concentration of gibberellic acid (GA) $(100 \mu \mathrm{M})$, abscisic acid (ABA) $(100 \mu \mathrm{M})$, methyl jasmonate (MeJA) $(100 \mu \mathrm{M})$ and salicylic acid (SA) $(500 \mu \mathrm{M})$ were prepared from stock solutions, after addition of wetting agent Tween-20 at $0.05 \%(\mathrm{v} / \mathrm{v})$ the individual hormone were sprayed on two weeks old rice leaves. For metals treatments, $\mathrm{FeSO}_{4}(7 \mathrm{mM}), \mathrm{CdCl}_{2}(0.5 \mathrm{mM}), \mathrm{K}_{2} \mathrm{Cr}_{2} \mathrm{O}_{7}$ (1 $\mathrm{mM})$, and $\mathrm{NiCl}_{2}(1 \mathrm{mM})$ were prepared from stock solutions and applied into fresh nutrient solution and as [24] with exception of phosphorus $(\mathrm{P})$ that prevents precipitation of lead $(\mathrm{Pb})$ [25]. The whole leaf blades from the treated two-week-old rice plants were harvested at $0 \mathrm{~h}$, $3 \mathrm{~h}, 6 \mathrm{~h}, 12 \mathrm{~h}$ and $24 \mathrm{~h}$ time intervals after treatments. Rice plants were allowed to grow in normal condition (day/ night temperature cycle of $32^{\circ} \mathrm{C} / 26^{\circ} \mathrm{C}, 16 \mathrm{~h} / 8 \mathrm{~h}$ photo-period with $800 \mu \mathrm{mol} \mathrm{m}^{-2} \mathrm{~s}^{-1}$ light intensity and $60 \%$ humidity), and the different plant organs at various developmental stages (namely seedling, tillering, booting and heading stages) were collected for the analysis of tissue-specific expression. The samples were immediately frozen in liquid nitrogen and stored at $-80^{\circ} \mathrm{C}$ until for further analysis.

\section{Quantitative PCR analysis}

The total RNA was extracted from all the samples by using the cetyltrimethylammonium bromide (CTAB) method [26]. The samples were run on $2 \%$ agarose gels to examine the intensity of ribosomal RNA (rRNA) 
bands, degraded products, and gDNA contamination. The residual genomic DNA was removed by treating the RNA samples with RNase-free DNase. The cDNA was synthesized through the PrimeScript RT Reagent Kit with gDNA Eraser (Takara Bio, Shiga, Japan) following the manufacturer's instructions. All the primers were designed from rice $B B X$ sequences for real-time PCR using primer 6.0 (Additional file 1: Table S3). Each primer pair was examined through standard RT-PCR to confirm the size of the amplified product through 1\% agarose gel electrophoresis. Real-time PCR was carried out in an iCycler iQ Real-Time PCR Detection System (Bio-Rad). Each reaction consisted of $5 \mu \mathrm{l}$ SYBR Premix ExTaq (Takara, Kyoto, Japan), $2 \mu \mathrm{l}$ cDNA samples, and $0.5 \mu \mathrm{l}$ of

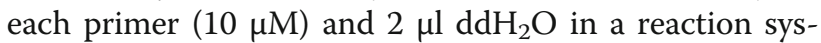
tem of $10 \mu \mathrm{l}$. The thermal cycle was as follows: $95^{\circ} \mathrm{C}$ for $3 \mathrm{~min}$, followed by 40 cycles at $94^{\circ} \mathrm{C}$ for $15 \mathrm{~s}, 62^{\circ} \mathrm{C}$ for 20 $\mathrm{s}$, and $72{ }^{\circ} \mathrm{C}$ for $20 \mathrm{~s}$. Melting-curve analysis was performed directly after real-time PCR to verify the presence of gene-specific PCR products. This analysis was done by $94^{\circ} \mathrm{C}$ for $15 \mathrm{~s}$, followed by a constant increase from 60 to $95^{\circ} \mathrm{C}$ at a $2 \%$ ramp rate. The rice actin gene (OsActin1, Gene ID: KC140126) was used as an internal control and served as a standard gene for normalizing all mRNA expression levels. The relative amount of template present in each PCR amplification mixture was evaluated by using the $2-\Delta \Delta \mathrm{Ct}$ method.

\section{Statistical analysis}

The data underwent an analysis of variance. The means and standard deviation of three replications for all the treatments were compared by the least significant difference (LSD) test at the 5\% level using the SPSS 11.5 software package (SPSS, Chicago, IL, USA). Graphs were designed using Origin 7.5 (Microcal Software Inc., Northampton, MA, USA).

\section{Results}

Identification, Classification, and Annotation of $B B X$ Family Members

The Arabidopsis $B B X$ genes were used as quarries sequences against the Hidden Markov Model (HMM) algorithm [27] to retrieve and characterize the $B B X$ gene family members in five Poaceae species. A total of $131 B B X$ genes were identified in the studied five Poaceae species. The number of $B B X$ genes members were diverse among these plants such as 36, 30, 24, 22 and 19 BBX genes from maize (Zea mays), rice (Oryza sativa), Sorghum (Sorghum bicolor), stiff brome (Brachypodium distachyon) and Millet (Setaria italica), respectively (Table 1). The potential domains of $B B X$ gene family were confirmed through the conserved domain database, Pfam and SMART databases and structural integrity of these domains were drawn by Web Logo and EXPASY-PROSITE. All the putative $B B X$ members lack transmembrane segment except $Z m B B X 30$ (Additional file 1: Figure S1). Moreover, the physiochemical characteristics and amino acid sequence of $B B X$ members were studied through EXPASY PROTOPARAM (http://www.expasy.org/ tools/protparam.html) online tool (Additional file 1: Table $\mathrm{S} 1)$. The assumed length of the BBX proteins and molecular weights vary widely, ranging from 9.51 (OsBBX20) to 52.89 $\mathrm{kD}$ (SbBBX10). The maximum number of Poaceae BBX proteins was acidic in nature according to their isoelectric point, which was lower than seven. However, the isoelectric point of some $B B X$ members (OsBBX15, OsBBX20, OsBBX21, ZmBBX17, ZmBBX19, ZmBBX24, BdBBX16, $S b B B X 6$, and $S b B B X 11)$ was greater than seven, indicating that they are alkaline proteins in nature. The present study divided the majority of Poaceae $B B X$ genes into unstable proteins because the instability index of most of the genes of this family was greater than 40 . However, the instability index of $B d B B X 20, O s B B X 12$, and $O s B B X 20$ were less than 40 , and they corresponded to stable proteins. All the BBX proteins were found to be hydrophilic except OsBBX25 based on their GRAV value. $Z m B B X 9$ showed high negative and positive charge residues. Based on a total number of atoms, $S b B B X 10$ contained the highest number of atoms (7281), followed by $Z m B B X 3$ (7200). OsBBX20 was the smallest protein (1299) on the basis of atom compositions. This investigation found that $68 \mathrm{BBX}$ proteins were located on the sense strand, and the remaining $63 \mathrm{BBX}$ proteins were found on the antisense strand. The GC content of the majority studied $B B X$ was above $60 \%$. Furthermore, the aliphatic index values ranged from lowest (39.91) (BdBBX11) to 78.93 (SbBBX11). The major amino acid of the BBX proteins is Ala, followed by Ser, while other most abundant amino acids are Pro, Asp, Asn, or Thr, varied depending on the particular BBX protein (Additional file 1: Table S1).

\section{Systematic Evolutionary Relationship, Gene Structural Diversity, and Motif Analysis}

We found four different classes of BBX proteins on the basis of domain organization; BBXs containing one B-box domain, BBXs having two B-boxes domains, BBXs possessing one B-box and additional CCT domain, and BBXs with two B-boxes and additional CCT domain (Table 2). The homologs of $B B X$ genes from six different species were selected for the multiple sequence alignments and phylogenetic relationships analysis to study the evolutionary phylogenetic relationships and functional divergence among $B B X$ genes (Fig. 1). We constructed an unrooted maximum-likelihood phylogenetic tree using MEGA 6.06 Software to investigate the evolutionary relationship. The present study clustered the $B B X$ genes into five well-conserved subfamilies based on the difference of protein topological structure with high bootstrap support (Fig. 1). The phylogenetic tree divided the $B B X$ from five Poaceae plants (maize, rice, sorghum, 
Table 1 Nomenclature, identification, chromosomal location, CDS, and peptide length and weight of BBX gene family in Poaceae species

\begin{tabular}{|c|c|c|c|c|c|c|c|}
\hline Name & Id & Location & Genomic & CDS & Protein & Strand & GC\% \\
\hline$\overline{Z m B B X 1}$ & Zm00001d029149 & Chr1: 60531179-60535397 & 4219 & 1191 & 397 & + & 63.0 \\
\hline ZmBBX2 & Zm00001d031662 & Chr1: 197918030-197921539 & 3510 & 1467 & 489 & - & 67.7 \\
\hline ZmBBX3 & Zm00001d033719 & Chr1: 272190270-272192279 & 2010 & 1413 & 471 & + & 67.2 \\
\hline ZmBBX4 & Zm00001d002806 & Chr2: 23197620-23201256 & 3636 & 774 & 258 & - & 68.2 \\
\hline ZmBBX5 & Zm00001d003162 & Chr2: 34080161-34081769 & 1608 & 960 & 320 & - & 72.0 \\
\hline ZmBBX6 & Zm00001d006198 & Chr2: 201392406-201400007 & 7601 & 762 & 254 & + & 48.9 \\
\hline ZmBBX7 & Zm00001d007107 & Chr2: 222393488-222401395 & 7907 & 1227 & 409 & - & 70.8 \\
\hline ZmBBX8 & Zm00001d039437 & Chr3: 4316527-4318099 & 1572 & 1077 & 359 & + & 74.3 \\
\hline ZmBBX9 & Zm00001d049347 & Chr4: 27315744-27316583 & 839 & 837 & 279 & - & 72.3 \\
\hline $\mathrm{ZmBBX} 10$ & Zm00001d051018 & Chr4: 137141478-137142700 & 1222 & 759 & 253 & - & 69.0 \\
\hline ZmBBX11 & Zm00001d051047 & Chr4: 138655083-138656121 & 1038 & 942 & 314 & + & 75.3 \\
\hline $\mathrm{ZmBBX} 12$ & Zm00001d051309 & Chr4: $152948011-152949738$ & 1727 & 831 & 277 & + & 71.7 \\
\hline ZmBBX13 & Zm00001d051610 & Chr4: 164536434-164541204 & 4770 & 1410 & 470 & - & 46.7 \\
\hline ZmBBX14 & Zm00001d051684 & Chr4: 166653210-166659390 & 6180 & 1218 & 406 & + & 46.7 \\
\hline ZmBBX15 & Zm00001d013443 & Chr5: 11710780-11712488 & 1708 & 1239 & 413 & - & 68.1 \\
\hline ZmBBX16 & Zm00001d014765 & Chr5: 62282614-62284983 & 2369 & 1065 & 355 & - & 69.5 \\
\hline ZmBBX17 & Zm00001d015434 & Chr5: 89924027-89924707 & 680 & 678 & 226 & - & 79.8 \\
\hline ZmBBX18 & Zm00001d017176 & Chr5: 188032923-188034708 & 1785 & 1005 & 335 & + & 74.5 \\
\hline ZmBBX19 & Zm00001d017412 & Chr5: 195189894-195191367 & 1473 & 426 & 142 & + & 72.4 \\
\hline ZmBBX20 & Zm00001d017885 & Chr5: 209614260-209620254 & 5994 & 1383 & 461 & + & 48.1 \\
\hline ZmBBX21 & Zm00001d017939 & Chr5: 210407150-210409118 & 1968 & 1395 & 465 & - & 67.5 \\
\hline ZmBBX22 & Zm00001d036214 & Chr6: 77322780-77327461 & 4681 & 1158 & 386 & + & 50.8 \\
\hline ZmBBX23 & Zm00001d036418 & Chr6: 87645024-87646346 & 1322 & 807 & 269 & + & 72.0 \\
\hline ZmBBX24 & Zm00001d036676 & Chr6: 97378208-97378813 & 605 & 603 & 201 & - & 81.1 \\
\hline ZmBBX25 & Zm00001d037327 & Chr6: 121369894-121371711 & 1817 & 1356 & 452 & + & 69.5 \\
\hline ZmBBX26 & Zm00001d037735 & Chr6: 135623653-135625596 & 1943 & 966 & 322 & - & 72.6 \\
\hline ZmBBX27 & Zm00001d021278 & Chr7: 147632776-147636889 & 4113 & 618 & 206 & + & 50.7 \\
\hline ZmBBX28 & Zm00001d045323 & Chr9: 18935792-18946869 & 1077 & 1266 & 422 & - & 51.8 \\
\hline ZmBBX29 & Zm00001d045661 & Chr9: 32056769-32058471 & 1702 & 1368 & 456 & + & 68.1 \\
\hline ZmBBX30 & Zm00001d045735 & Chr9: 36009335-36013889 & 4554 & 1284 & 428 & + & 61.1 \\
\hline ZmBBX31 & Zm00001d045804 & Chr9: 40102185-40108023 & 5838 & 1308 & 436 & - & 43.9 \\
\hline ZmBBX32 & Zm00001d046925 & Chr9: 111032442-111034142 & 1700 & 1092 & 364 & + & 70.5 \\
\hline ZmBBX33 & Zm00001d024200 & Chr10: 55274175-55275011 & 836 & 834 & 278 & - & 72.4 \\
\hline ZmBBX34 & Zm00001d024213 & Chr10: 56996605-56997874 & 1269 & 780 & 260 & - & 71.7 \\
\hline ZmBBX35 & Zm00001d025770 & Chr10: 129045322-129046879 & 1557 & 969 & 323 & + & 72.6 \\
\hline ZmBBX36 & Zm00001d025957 & Ch10: $134741085-134742626$ & 1541 & 768 & 256 & + & 68.0 \\
\hline OsBBX1 & Os01g0202500 & chr01:5639835..5641475 & 1440 & 1065 & 355 & + & 72.4 \\
\hline OsBBX2 & Os02g0176000 & chr02:4150302..4150970 & 669 & 669 & 223 & - & 77.4 \\
\hline OsBBX3 & Os02g0178100 & chr02:4315391..4316956 & 1370 & 996 & 332 & + & 73.5 \\
\hline OsBBX4 & Os02g0606200 & chr02:23759252..23760433 & 1078 & 816 & 272 & + & 71.3 \\
\hline OsBBX5 & Os02g0610500 & chr02:23989803..23991271 & 1388 & 999 & 333 & + & 73.7 \\
\hline OsBBX6 & Os02g0646200 & chr02:26027785..26029488 & 1198 & 810 & 270 & + & 71.3 \\
\hline OsBBX7 & Os02g0724000 & chr02:30094300..30099072 & 2023 & 1224 & 408 & + & 46.2 \\
\hline
\end{tabular}


Table 1 Nomenclature, identification, chromosomal location, CDS, and peptide length and weight of BBX gene family in Poaceae species (Continued)

\begin{tabular}{|c|c|c|c|c|c|c|c|}
\hline Name & Id & Location & Genomic & CDS & Protein & Strand & GC\% \\
\hline$\overline{\text { OsBBX8 }}$ & Os02g0731700 & chr02:30473739..30475800 & 1469 & 1044 & 348 & - & 64.4 \\
\hline OsBBX9 & Os03g0351100 & chr03:13153018..13155544 & 1638 & 1212 & 404 & + & 61.5 \\
\hline OsBBX10 & Os03g0711100 & chr03:28686958..28689501 & 1840 & 1266 & 422 & + & 73.6 \\
\hline OsBBX11 & Os04g0493000 & chr04:24648004..24648863 & 765 & 555 & 185 & - & 66.3 \\
\hline OsBBX12 & Os04g0497700 & chr04:24889983..24891483 & 1394 & 1002 & 334 & + & 74.9 \\
\hline OsBBX13 & Os04g0540200 & chr04:27027267..27029421 & 1220 & 753 & 251 & + & 70.3 \\
\hline OsBBX14 & Os05g0204600 & chr05:6514746..6517280 & 2058 & 1137 & 379 & + & 72.2 \\
\hline OsBBX15 & Os06g0103000 & chr06:209204..210107 & 904 & 672 & 223 & + & 56.8 \\
\hline OsBBX16 & Os06g0152200 & chr06:2695460..2699468 & 1449 & 1083 & 361 & + & 52.1 \\
\hline OsBBX17 & Os06g0264200 & chr06:8704897..8706342 & 1446 & 1446 & 482 & + & 71.7 \\
\hline OsBBX18 & Os06g0275000 & chr06:9336376..9338569 & 1557 & 1188 & 396 & + & 58.0 \\
\hline OsBBX19 & Os06g0298200 & chr06:11070174..111076691 & 2201 & 1227 & 409 & - & 47.3 \\
\hline OsBBX20 & Os06g0654900 & chr06:26843118..26843680 & 563 & 246 & 82 & - & 69.1 \\
\hline OsBBX21 & Os06g0661200 & chr06:27253336..27254108 & 773 & 726 & 242 & + & 78.0 \\
\hline OsBBX22 & Os06g0713000 & chr06:30196103..30197569 & 1380 & 927 & 309 & + & 74.0 \\
\hline OsBBX23 & Os07g0667300 & chr07:28184879..28187843 & 1547 & 1143 & 381 & - & 64.4 \\
\hline OsBBX24 & Os08g0178800 & chr08:4610545..4612918 & 1402 & 846 & 282 & - & 73.0 \\
\hline OsBBX25 & Os08g0249000 & chr08:9098485..9099878 & 1025 & 543 & 181 & + & 76.2 \\
\hline OsBBX26 & Os08g0536300 & chr08:26792942..26797114 & 1891 & 1467 & 489 & - & 63.8 \\
\hline OsBBX27 & Os09g0240200 & chr09:3048085..3064471 & 1362 & 1008 & 336 & - & 66.7 \\
\hline OsBBX28 & Os09g0509700 & chr09:19783524..19786772 & 1813 & 1296 & 432 & - & 64.3 \\
\hline OsBBX29 & Os09g0527900 & chr09:20646416..20649984 & 1248 & 636 & 212 & - & 49.0 \\
\hline OsBBX30 & Os12g0209200 & chr12:5699790..5702413 & 792 & 633 & 211 & + & 71.5 \\
\hline SbBBX1 & Sb01g010420 & Chr1: 9244438-9245891 & 2454 & 1260 & 420 & - & 59.0 \\
\hline SbBBX2 & Sb01g035400 & Chr1: 58949641-58952463 & 3823 & 1239 & 413 & - & 49.7 \\
\hline SbBBX3 & Sb02g030690 & Chr2: 65720371-65723158 & 3788 & 633 & 211 & + & 44.8 \\
\hline SbBBX4 & Sb02g042230 & Chr2: 75932483-75934993 & 3511 & 1239 & 413 & - & 53.0 \\
\hline SbBBX5 & Sb03g002510 & Chr3: 2287972-2290172 & 3201 & 1056 & 352 & - & 56.5 \\
\hline SbBBX6 & Sb04g003470 & Chr4: 3321373-3324297 & 3925 & 1458 & 486 & + & 49.6 \\
\hline SbBBX7 & Sb04g005250 & Chr4: 5107155-5108908 & 2754 & 1128 & 376 & + & 50.1 \\
\hline SbBBX6 & Sb04g025400 & Chr4: 55156181-55157415 & 2235 & 789 & 263 & - & 56.8 \\
\hline SbBBX7 & Sb04g025660 & Chr4: 55434304-55435890 & 2587 & 1008 & 336 & + & 56.3 \\
\hline SbBBX8 & Sb04g028920 & Chr4: 58992284-58994122 & 2839 & 1464 & 488 & + & 58.8 \\
\hline SbBBX9 & Sb04g029180 & Chr4: 59189963-59191466 & 2504 & 867 & 289 & + & 40.6 \\
\hline SbBBX12 & Sb04g029480 & Chr4: 59566100-59571089 & 5990 & 1218 & 406 & - & 45.9 \\
\hline SbBBX10 & Sb04g033440 & Chr4:63334637-63336646 & 3010 & 885 & 295 & - & 52.2 \\
\hline SbBBX11 & Sb06g021170 & Chr6: 50527547-50528989 & 2443 & 804 & 268 & - & 55.1 \\
\hline SbBBX12 & Sb06g021480 & Chr6:50736218-50737513 & 2296 & 987 & 329 & + & 60.2 \\
\hline SbBBX13 & Sb06g023960 & Chr6: 53024486-53026365 & 2880 & 792 & 264 & + & 52.0 \\
\hline SbBBX14 & Sb07g004973 & Chr7: 6615154-6617300 & 2869 & 813 & 271 & - & 52.2 \\
\hline SbBBX15 & Sb07g025940 & Chr7: 61088100-61092224 & 5125 & 1470 & 490 & + & 51.2 \\
\hline SbBBX16 & Sb08g006510 & Chr8: 10161992-10163814 & 2832 & 735 & 245 & + & 46.7 \\
\hline SbBBX17 & Sb09g006370 & Chr9: 9921800-9923032 & 2233 & 1008 & 336 & + & 63.3 \\
\hline
\end{tabular}


Table 1 Nomenclature, identification, chromosomal location, CDS, and peptide length and weight of BBX gene family in Poaceae species (Continued)

\begin{tabular}{|c|c|c|c|c|c|c|c|}
\hline Name & Id & Location & Genomic & CDS & Protein & Strand & GC\% \\
\hline SbBBX18 & Sb10g003680 & $3189527-3194818$ & 6292 & 1125 & 375 & + & $\overline{44.4}$ \\
\hline SbBBX19 & Sb10g009480 & Chr10: 10694648-10696491 & 2844 & 1422 & 474 & + & 57.0 \\
\hline SbBBX20 & Sb10g010050 & Chr10: 12275128-12276617 & 2490 & 1233 & 411 & + & 50.1 \\
\hline $\mathrm{SbBBX} 21$ & Sb10g010860 & Chr10: 14421774-14424602 & 3829 & 1218 & 406 & - & 39.8 \\
\hline SbBBX22 & Sb10g026060 & Chr10: 59584561-595898674 & 2801 & 1128 & 376 & - & 58.9 \\
\hline SbBBX23 & Sb10g029840 & Chr10: 55386280-55388080 & 2484 & 915 & 305 & - & 53.8 \\
\hline SbBBX24 & Sb10g002725 & Chr10: 59584561-595898674 & 2354 & 900 & 300 & - & 50.4 \\
\hline BdBBX1 & Bradi1g11310 & Chr1: 8356244-8358332 & 2089 & 1269 & 423 & + & 60.0 \\
\hline BdBBX2 & Bradi1g31280 & Chr1: 26748157-26749884 & 2928 & 1044 & 348 & + & 55.2 \\
\hline BdBBX3 & Bradi1g35030 & Chr1: 30557402-30558987 & 2789 & 867 & 289 & - & 53.4 \\
\hline BdBBX4 & Bradi1g43220 & Chr1: 40823689-40831526 & 5023 & 1134 & 378 & + & 40.9 \\
\hline BdBBX5 & Bradi1g43670 & Chr1: 41483191-41486564 & 4578 & 1308 & 436 & - & 40.2 \\
\hline BdBBX6 & Bradi1g43990 & Chr1: 41909639-41911589 & 3151 & 1101 & 367 & - & 57.3 \\
\hline BdBBX7 & Bradi1g49260 & Chr1: 48014263-48018866 & 5804 & 1134 & 378 & - & 44.4 \\
\hline $\mathrm{BdBBX} 8$ & Bradi1g62420 & Chr1: 61651141-61654384 & 4444 & 1056 & 352 & - & 54.1 \\
\hline BdBBX9 & Bradi2g06370 & Chr2: 4839844-4841420 & 2777 & 999 & 333 & + & 57.1 \\
\hline BdBBX10 & Bradi2g32900 & Chr2: 32830551-32831957 & 2607 & 1137 & 379 & - & 53.8 \\
\hline BdBBX11 & Bradi3g05800 & Chr3: 4124615-4126380 & 2966 & 693 & 231 & + & 55.9 \\
\hline $\mathrm{BdBBX} 12$ & Bradi3g15490 & Chr3: 13785466-13787484 & 3219 & 1470 & 490 & + & 48.8 \\
\hline BdBBX13 & Bradi3g41500 & Chr3: 43415799-43419719 & 5121 & 774 & 258 & - & 49.9 \\
\hline BdBBX14 & Bradi3g48180 & Chr3: 49677419-49678624 & 2405 & 1239 & 413 & - & 54.2 \\
\hline BdBBX15 & Bradi3g56260 & Chr3: 56219290-56221877 & 3788 & 1179 & 393 & + & 41.1 \\
\hline BdBBX16 & Bradi3g56490 & Chr3: 56348167-56352072 & 5106 & 1344 & 448 & - & 41.7 \\
\hline BdBBX17 & Bradi3g57000 & Chr3: 56695125-56696699 & 2345 & 633 & 211 & + & 49.4 \\
\hline BdBBX18 & Bradi4g35950 & Chr4: 41215132-41218558 & 4627 & 666 & 222 & + & 44.0 \\
\hline BdBBX19 & Bradi4g40250 & Chr4: 44678780-44680513 & 2934 & 801 & 267 & - & 45.1 \\
\hline BdBBX20 & Bradi5g14280 & Chr5: 17712355-17713552 & 3908 & 1023 & 341 & + & 48.4 \\
\hline BdBBX21 & Bradi5g14600 & Chr5: 18046925-18048712 & 2988 & 801 & 267 & + & 60.8 \\
\hline BdBBX22 & Bradi5g17080 & Chr5: 20311769-20313350 & 2782 & 801 & 267 & + & 53.5 \\
\hline SiBBX1 & Si017487m & Chr1: 6104121-6106008 & 3088 & 1158 & 386 & - & 74.8 \\
\hline SiBBX2 & Si018081m & Chr1: 32838588-32840495 & 3108 & 807 & 269 & + & 71.7 \\
\hline SiBBX3 & Si017374m & Chr1: 36700617-36705652 & 6236 & 1221 & 407 & + & 45.8 \\
\hline SiBBX4 & Si019803m & Chr1: $36911502-36913121$ & 2820 & 1065 & 355 & - & 45.6 \\
\hline SiBBX5 & Si017124m & Chr1: 37159623-37161711 & 3289 & 1395 & 465 & - & 70.8 \\
\hline SiBBX6 & Si030034m & Chr2: 26392218-26396291 & 5274 & 1218 & 406 & + & 47.5 \\
\hline SiBBX7 & Si031264m & Chr2: 38047130-38051532 & 5603 & 531 & 177 & + & 45.3 \\
\hline SiBBX8 & Si022650m & Chr3: 4644038-4645844 & 3007 & 978 & 326 & + & 73.9 \\
\hline SiBBX9 & Si024510m & Chr3: $6380462-6382407$ & 3146 & 654 & 218 & - & 71.3 \\
\hline SiBBX10 & Si006670m & Chr4: 5544882-5549372 & 5691 & 1134 & 378 & - & 53.3 \\
\hline SiBBX11 & Si006432m & Chr4: 11409189-114111174 & 3186 & 1335 & 445 & + & 72.1 \\
\hline SiBBX12 & Si006690m & Chr4: 31034615-31036532 & 3118 & 1116 & 372 & + & 70.0 \\
\hline SiBBX13 & Si006993m & Chr4: 39392572-39394067 & 2696 & 903 & 301 & - & 71.9 \\
\hline SiBBX14 & Si001636m & Chr5: 12649122-12651152 & 3231 & 1239 & 413 & + & 73.0 \\
\hline
\end{tabular}


Table 1 Nomenclature, identification, chromosomal location, CDS, and peptide length and weight of BBX gene family in Poaceae species (Continued)

\begin{tabular}{|c|c|c|c|c|c|c|c|}
\hline Name & Id & Location & Genomic & CDS & Protein & Strand & GC\% \\
\hline SiBBX16 & Si014382m & Chr6: 33985026-33988379 & 3354 & 705 & 235 & - & 49.0 \\
\hline SiBBX17 & Si014037m & Chr6: 33985026-33989297 & 5472 & 1008 & 336 & - & 49.0 \\
\hline SiBBX15 & Si010884m & Chr7: 23710294-23711500 & 2407 & 774 & 258 & - & 70.2 \\
\hline SiBBX16 & Si010592m & Chr7: 23922376-23923928 & 2753 & 978 & 326 & + & 74.2 \\
\hline SiBBX17 & Si010885m & Chr7: 26130036-26131926 & 3091 & 774 & 258 & + & 69.7 \\
\hline SiBBX18 & Si035937m & Chr9: 7441605-7443503 & 3099 & 1218 & 406 & - & 70.6 \\
\hline SiBBX19 & Si034611m & Chr9: 46372657-46376370 & 4914 & 1944 & 648 & - & 64.8 \\
\hline
\end{tabular}

stiff brome, and millet) and one model plant (Arabidopsis) into five subfamilies based on our analysis. Maximum numbers of $B B X$ genes containing only one B-box domain were found in subfamily II, IV and V. Most of $B B X$ genes with two B-boxes domains were clustered into subfamily $\mathrm{V}$ and IV. The third class of $B B X$ genes, containing one B-box and additional CCT domain were observed in subfamily I, II and III. Two B-box possessing genes with additional CCT domain were grouped to subfamily I and III. Furthermore, we also evaluated the Arabidopsis $B B X$ genes to study their phylogenetic relationship with Poaceae $B B X$ members. We found quite a similar clustering for Arabidopsis $B B X$ genes with Poaceae $B B X$ genes in this study (Fig. 1). Arabidopsis $B B X$ possessing only one B-box domain was detected in subfamily II and IV. Two B-boxes domains containing $A t B B X s$ were grouped into IV and V. AtBBXs with one B-box and additional CCT domain containing genes were detected in subfamily II, whereas two B-boxes and additional CCT possessing AtBBXs genes were noted in subfamily I and III.

The conservation of gene structure in a paralogous gene is sufficient to determine the evolutionary connection between introns in various circumstances; therefore, an exon-intron diagram of the $B B X$ genes members was constructed according to their genomic and coding sequences (Additional file 1: Figure S2). The exon-intron distribution of all the studied $B B X$ family members was investigated through GSDS online software. The range of a number of introns was from one to seventeen $(\mathrm{ZmBBX} 28)$ in this study. However, we also identified some $B B X$ members without of intron, they comprised only of the exon. For instance, $Z m B B X 9, Z m B B X 17, Z m B B X 24$, and $Z m B B X 33$ genes have the only exon in maize. In rice, $O s B B X 2$ and $O s B B X 25$ were found without of intron. However, without of intron genes were not found in sorghum, stiff brome, and millet.

Furthermore, all the BBX proteins were run on MEME tool to investigate the motifs (Additional file 1: Figure S2). MEME analysis found a total of 10 motifs and was named 1-10. Based on width, Motif-7 was the largest motif, whereas next spots were held by motif- 8 and motif-2
(Additional file 1: Table S2). We observed that motif-2 was present in 126 out of $131 B B X$ members, followed by the shortest motif, named motif-6 (115 $B B X$ members). The longest motif (motif-7) was only found in $10 B B X$ members. Similarly, each motif- 8 and 9 were found in 11 $B B X$ members.

\section{Chromosomal Location, Multiple Alignments and Gene Duplication of $B B X$ Genes}

The chromosome location and annotation information of the $B B X$ genes showed that $B B X$ genes are unevenly distributed on the chromosomes in the genome of the studied species (Additional file 1: Figure S3). In maize, all the $36 \mathrm{ZmBBX}$ genes were found to be distributed on the 10 chromosomes, except for chromosomes 8 (Additional file 1: Figure S3A). However, the number of $Z m B B X$ genes varied widely on each chromosome. A high number of $Z m B B X$ genes (7) was localized on chromosome no. 5, whereas $6,5,5,4,4,3,1$ and $1 \mathrm{ZmBBX}$ members were identified on chromosomes 4, 6, 9, 2, 10, 1, 3 and 7 in the maize genome, respectively. In rice, all $30 O s B B X$ genes are distributed on chromosomes 1-9: 8 OsBBXs are located on chromosome 6,7 rice $B B X$ members were detected on chromosomes 2, 3 OsBBX genes on each chromosome 4, 8 and 9, 2 OsBBX genes were found on chromosome 3, while 1 each on chromosomes 1, 5 and 7 (Additional file 1: Figure S3B). In sorghum, $S b B B X$ genes were found to be distributed on all the chromosomes except chromosome 5: $8 \mathrm{SbBBXs}$ were found on chromosome 4, $6 \mathrm{SbBBXs}$ on chromosome 10, $3 \mathrm{SbBBX}$ s were detected on chromosome 6, $2 S b B B X$ members are present on each 1, 2 and 7, while one each on chromosomes 3, 8 and 9 (Additional file 1: Figure S3C). All the $B d B B X$ genes member are distributed on all chromosomes in stiff brome genome. A maximum number of $B d B B X$ genes are localized on chromosome $1(8 B d B B X s)$ and 3 (7 $B d B B X s$ ). Remaining $B d B B X$ members are distributed as: $3 B d B B X s$ on chromosome 5 , while $2 B d B B X$ genes are located on each chromosome 2 and 4. SiBBX genes were detected on all chromosome expect on chromosome 8 (Additional file 1: Figure S3D). The number of $B B X$ genes 
Table 2 Structures of the BBX proteins. The length and order of the domains represent their actual location within each protein

\begin{tabular}{|c|c|c|c|c|c|}
\hline Name & Domains & BBX1 & BBX2 & CCT & Structure \\
\hline 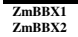 & $\begin{array}{l}I \mathrm{IBBX+CCT} \\
\mathrm{IBBX}+\mathrm{CCCT}\end{array}$ & $\begin{array}{l}50.92 \\
15.58 \\
58\end{array}$ & & $\begin{array}{l}352-395 \\
444-478 \\
\end{array}$ & 81 \\
\hline $\begin{array}{l}\mathrm{ZmBBB3} \\
\mathrm{ZmBBX} 4\end{array}$ & $\begin{array}{l}1 \mathrm{BBX}+\mathrm{CCCT} \\
\mathrm{C} \mathrm{BX}\end{array}$ & 年-62 & 60-107 & & \\
\hline $\begin{array}{l}\text { ZmBBX5 } \\
\text { ZmBBX6 }\end{array}$ & $\begin{array}{l}2 \mathrm{BBX}+\mathrm{CCCT} \\
\mathrm{BBBX}\end{array}$ & 20-135 20.6135 & 57-101 & $236-278$ & cet \\
\hline 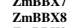 & $\begin{array}{l}\text { IBBXX+CCT } \\
2 \mathrm{BBX}\end{array}$ & & 58-100 & 364-407 & cer \\
\hline $\begin{array}{l}\mathrm{ZmBXX9} \\
\mathrm{ZmBX} 10\end{array}$ & 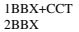 & $\begin{array}{l}26-57 \\
5.47\end{array}$ & 55.98 & 179-216 & तect \\
\hline $\begin{array}{l}\mathrm{ZmBBB11} \\
\mathrm{ZmBX} 12\end{array}$ & 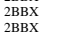 & $\begin{array}{l}22-63 \\
2.47\end{array}$ & $\begin{array}{l}238-280 \\
58-101\end{array}$ & & \\
\hline 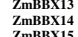 & 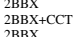 & 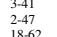 & 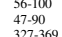 & 349-392 & नत्टा \\
\hline $\begin{array}{l}-1 \\
\mathrm{ZmBBP16}\end{array}$ & 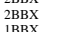 & 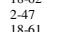 & 58-105 & & \\
\hline 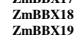 & & $\begin{array}{l}28-69 \\
2-47\end{array}$ & $\begin{array}{l}257-299 \\
64-108\end{array}$ & & 38 \\
\hline $\begin{array}{l}\mathrm{ZmBBX20} \\
\mathrm{ZmBBX} 21\end{array}$ & IIBBX+CCT & $\begin{array}{l}64.107 \\
19-61\end{array}$ & & 404-447 & त्वा \\
\hline 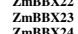 & $\begin{array}{l}2 \\
2 \mathrm{BBX} X \\
2 \mathrm{BPX}\end{array}$ & 年-41 & $\begin{array}{l}62-177 \\
58-102\end{array}$ & & \\
\hline $\begin{array}{l}\mathrm{ZmBB \times 25} \\
\mathrm{ZmBB \times 26}\end{array}$ & 要BXX+CCT & $\begin{array}{l}24.66 \\
24.47\end{array}$ & 50.05 & $397-439$ & तect \\
\hline 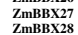 & 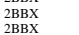 & $\begin{array}{l}5.45 \\
5.47\end{array}$ & $\begin{array}{l}52 \cdot 96 \\
102-2139\end{array}$ & & \\
\hline 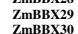 & 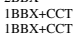 & $\begin{array}{l}22-66 \\
6.3-107\end{array}$ & & $\begin{array}{l}401-443 \\
357-400 \\
4\end{array}$ & $\begin{array}{ll}\text { Cect } \\
\text { CCT }\end{array}$ \\
\hline $\begin{array}{l}\mathrm{ZmBBX31} \\
\mathrm{ZmBBX322}\end{array}$ & $\begin{array}{l}2 \mathrm{BBX+CCCT} \\
2 \mathrm{BBX}+C \mathrm{CCT}\end{array}$ & $\begin{array}{l}4.47 \\
12.55 \\
-10\end{array}$ & $\begin{array}{l}47.90 \\
51-98\end{array}$ & $\begin{array}{l}379-422 \\
286-328\end{array}$ & Er \\
\hline 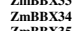 & 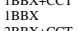 & 22-63 & & 203.229 & $\sqrt{\bar{\sigma}}$ \\
\hline 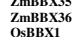 & 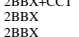 & $\begin{array}{l}20-61 \\
2.47 \\
1-31\end{array}$ & $\begin{array}{l}57-104 \\
56-103 \\
59-101\end{array}$ & $236-278$ & Н СCT \\
\hline $\begin{array}{lll}\mathrm{BBBX} 2 \\
\mathrm{BBB \times 3}\end{array}$ & IBBX & $\begin{array}{l}\begin{array}{l}21.56 \\
39-86\end{array} \\
3\end{array}$ & & 263-305 & Fत्वर \\
\hline $\begin{array}{l}\text { BBX4 } \\
\text { BBX55 }\end{array}$ & 2 & $\begin{array}{l}4.47 .72 \\
27.2\end{array}$ & 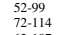 & 261-303 & Hect \\
\hline 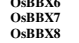 & $\begin{array}{l}2 \\
2 B B X+C C C T \\
18 B X+C C T\end{array}$ & $\begin{array}{l}2.47 \\
45-62 \\
15-62\end{array}$ & 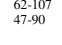 & $\begin{array}{l}372-393 \\
358-402\end{array}$ & $\begin{array}{ll}\mathrm{CET} \\
\overline{C E T}\end{array}$ \\
\hline 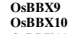 & $\begin{array}{l}1 \mathrm{BBXX+CCT} \\
\mathrm{BBB}+\mathrm{CCT}\end{array}$ & $\begin{array}{l}38-66 \\
15-68\end{array}$ & & $\begin{array}{l}358.401 \\
338-402 \\
-10\end{array}$ & |cet \\
\hline $\begin{array}{l}\text { DosBX11 } \\
\text { OsBBX12 } \\
\text { OsB }\end{array}$ & 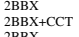 & $\begin{array}{l}4.47 \\
17-61\end{array}$ & 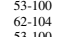 & 239-383 & - \\
\hline 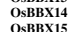 & $\begin{array}{l}2 \mathrm{BBA} \\
2 \mathrm{BBX}\end{array}$ & $36-83$ & $102-149$ & $164-207$ & Be \\
\hline 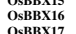 & 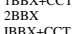 & $\begin{array}{l}4.47 \\
4.6 .63 \\
4\end{array}-17$ & 61-108 & $104-207$ & Bes \\
\hline $\begin{array}{l}\text { OsBBX18 } \\
\text { OsBBX19 }\end{array}$ & $\begin{array}{l}2 \mathrm{BBX+CCT} \\
2 \mathrm{BBX} C \mathrm{CCCT}\end{array}$ & $\begin{array}{l}30-77 \\
1.47\end{array}$ & $\begin{array}{c}78-116 \\
48-90\end{array}$ & $\begin{array}{l}326-370 \\
351.395 \\
3\end{array}$ & CT \\
\hline 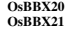 & $\begin{array}{l}2 \mathrm{BBX}+\mathrm{CCT} \\
\mathrm{BBBX}\end{array}$ & $\begin{array}{l}10.50 \\
3.49 \\
3\end{array}$ & $51-98$ & $295-339$ & CCT \\
\hline $\begin{array}{l}\text { OsBBX22 } \\
\text { OsBBX23 }\end{array}$ & 尊BX+CCT & $\begin{array}{l}1.47 \\
19-66\end{array}-2$ & $61-108$ & $337-380$ & तect \\
\hline 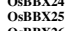 & 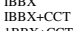 & 13.59 & & $435-479$ & ला \\
\hline 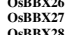 & 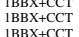 & $\begin{array}{l}13.60 \\
1.57 \\
1.48\end{array}$ & & 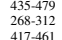 & 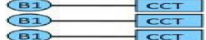 \\
\hline 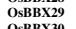 & 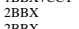 & $\begin{array}{l}1.47 \\
5.47\end{array}$ & $\begin{array}{l}51-96 \\
61-108\end{array}-100$ & & $8=$ \\
\hline 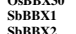 & 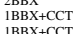 & 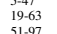 & & $\begin{array}{l}337-379 \\
36441\end{array}$ & $\sqrt{\mathrm{Cat}}$ \\
\hline $\begin{array}{l}\mathrm{SbBB \times 3} \\
\mathrm{S} \mathrm{sBB \times 4}\end{array}$ & $\begin{array}{l}1 \mathrm{PBX+CCT} \\
\mathrm{IBBX}+\mathrm{CCCT}\end{array}$ & 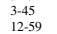 & & $\begin{array}{ll}55-96 \\
56 .-410 \\
3670\end{array}$ & 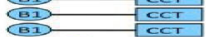 \\
\hline :BX5 & $\begin{array}{l}2 \mathrm{BBX} \\
\mathrm{BBBX}+\mathrm{CCT}\end{array}$ & 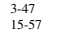 & 58-100 & $286-324$ & (3) \\
\hline BaX7 & $\begin{array}{l}2 \mathrm{BBX} \\
\mathrm{BBB}+\mathrm{CCCT}\end{array}$ & $\begin{array}{l}5-47 \\
24-65\end{array}$ & 52-98 & $258-300$ & नत्वा \\
\hline $\begin{array}{l}\text { SbbBX9 } \\
\text { SbBBX10 } \\
\text { SbBBXE10 }\end{array}$ & 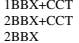 & $\begin{array}{l}22-64 \\
2.47 \\
2.47\end{array}$ & $\begin{array}{l}47-90 \\
66-113\end{array}$ & $\begin{array}{l}428-471 \\
371-392 \\
3\end{array}$ & 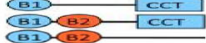 \\
\hline 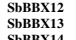 & 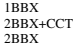 & $\begin{array}{l}61-104 \\
20-50 \\
2.47\end{array}$ & $\begin{array}{l}57-104 \\
60-107\end{array}$ & 242-284 & न्त्र \\
\hline 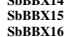 & 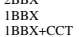 & $\begin{array}{l}19-65 \\
18-62 \\
-105\end{array}$ & & $438-480$ & त्वा \\
\hline 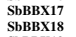 & 2 & $\begin{array}{l}5.48 \\
2.47 \\
\end{array}$ & 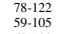 & & te \\
\hline $\begin{array}{l}\text { SbBBXX19 } \\
\text { SbBBX20 }\end{array}$ & I & $\begin{array}{l}53-67 \\
23-67\end{array}$ & 63-108 $>2$ & $418-460$ & $\mathrm{CCT}_{\mathrm{C}}$ \\
\hline $\begin{array}{l}\mathrm{SbBBX21} \\
\mathrm{SbBBX22}\end{array}$ & 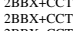 & $\begin{array}{l}33.77 \\
4.47 \\
.457\end{array}-3$ & $\begin{array}{l}76-120 \\
47-30 \\
7\end{array}$ & 371-3923 329 & $5 \frac{18}{182}$ \\
\hline 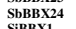 & 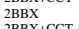 & $\begin{array}{l}1.47 \\
1.258\end{array}$ & 62-102 & 290.332 & 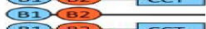 \\
\hline $\mathrm{BX} 2$ & 年 & $\begin{array}{l}2-47 \\
2.47 \\
-10\end{array}$ & 66-112 & & - ect \\
\hline $\begin{array}{l}S \text { SinBXX3 } \\
\text { SiBBX4 }\end{array}$ & I & 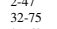 & & $\begin{array}{l}3253-328 \\
285\end{array}$ & $\begin{array}{cr}c T \\
c T\end{array}$ \\
\hline B. & 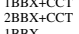 & $\begin{array}{l}21.63 \\
4.27 \\
.263\end{array}-3$. & $47-90$ & $\begin{array}{l}40-447 \\
371-392\end{array}$ & 1) - $1 \mathrm{CCT}$ \\
\hline 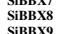 & & 2.47 & 63-109 & & 02 \\
\hline $\begin{array}{l}-10 \\
\text { SiBBXX10 }\end{array}$ & 2BBX & & $66-111$ & & \\
\hline $\begin{array}{l}\text { SibBX11 } \\
\text { SiBBX12 }\end{array}$ & $\begin{array}{l}1 \mathrm{BBX+CCT} \\
2 \mathrm{BBX}+\mathrm{CCT}\end{array}$ & ${ }_{12.56}^{24.56}$ & 52.99 & $\begin{array}{l}390-32 \\
297-339 \\
293\end{array}$ & BI \\
\hline $\begin{array}{l}\text { SiBBXX13 } \\
\text { SiBBX14 }\end{array}$ & ${ }_{2 \mathrm{BBX}}^{2 \mathrm{BBX}}$ & $\begin{array}{l}3.479 \\
84+129\end{array}-12$ & $\begin{array}{l}63-106 \\
135-182\end{array}$ & & 382 \\
\hline $\begin{array}{l}\text { SiBBXX15 } \\
\text { SiBB16 }\end{array}$ & 2 & $\begin{array}{l}50-61 \\
20-61\end{array}$ & $\begin{array}{l}57-100 \\
57-104\end{array}$ & $238-280$ & $5(182)$ - त्Cा \\
\hline 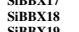 & I & $\begin{array}{l}18-62 \\
182\end{array}$ & & 322-363 & नar \\
\hline 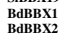 & 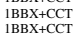 & $\begin{array}{l}1458 \\
1.498 \\
5.98\end{array}$ & & $\begin{array}{l}352-394 \\
272-315 \\
215\end{array}$ & त्ता \\
\hline 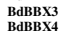 & $2 \mathrm{CBX}$ & $\begin{array}{l}2.470 \\
247\end{array}$ & $\begin{array}{c}58-105 \\
47-88\end{array}$ & $357-388$ & 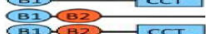 \\
\hline $\begin{array}{l}\text { BdBBX5 } \\
\text { BdBBX66 }\end{array}$ & 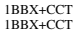 & 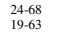 & & 309-352 3024 & $\begin{array}{lll}15 & \mathrm{ccr} \\
1 & \mathrm{ccr}\end{array}$ \\
\hline 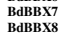 & $\begin{array}{l}2 \mathrm{BBX} \\
\mathrm{IBBX}+\mathrm{CCT}\end{array}$ & $\begin{array}{l}5.47 \\
20-62 \\
5\end{array}-2$ & 64-107 & 331-374 & B15 (102) तCT \\
\hline $\begin{array}{l}\text { BdBBX9 } \\
\text { BdBBX10 }\end{array}$ & 2BBX & $\begin{array}{l}2.34 \\
2.47 \\
2.47\end{array}$ & $\begin{array}{l}64-106 \\
655108 \\
60.324\end{array}$ & & 52 \\
\hline 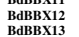 & 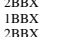 & $\begin{array}{l}18.59 \\
10.53\end{array}-10$ & $437-480$ & & (8) \\
\hline $\begin{array}{l}\text { BabBX14 } \\
\text { BdBBX15 }\end{array}$ & $\begin{array}{l}2 \mathrm{BBX} \\
2 \mathrm{BBX}+\mathrm{CCT}\end{array}$ & $\begin{array}{l}5.47 \\
70-114 \\
5\end{array}$ & $\begin{array}{l}53.99 \\
110-157\end{array}-15$ & 344-387 & 1802 CCT \\
\hline $\begin{array}{l}\text { BdBBXX16 } \\
\text { BdBBX17 }\end{array}$ & 甚BBX+CCCT & 24.68 & & $\begin{array}{l}359-719 \\
395-438\end{array}$ & 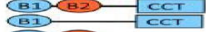 \\
\hline $\begin{array}{l}3.8 \times 18 \\
B \times 19\end{array}$ & 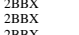 & $\begin{array}{l}5.45 \\
5.47 \\
5.47\end{array}$ & 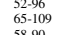 & & (11) \\
\hline 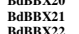 & 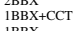 & 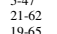 & & 243-285 & $15-$ aer \\
\hline
\end{tabular}




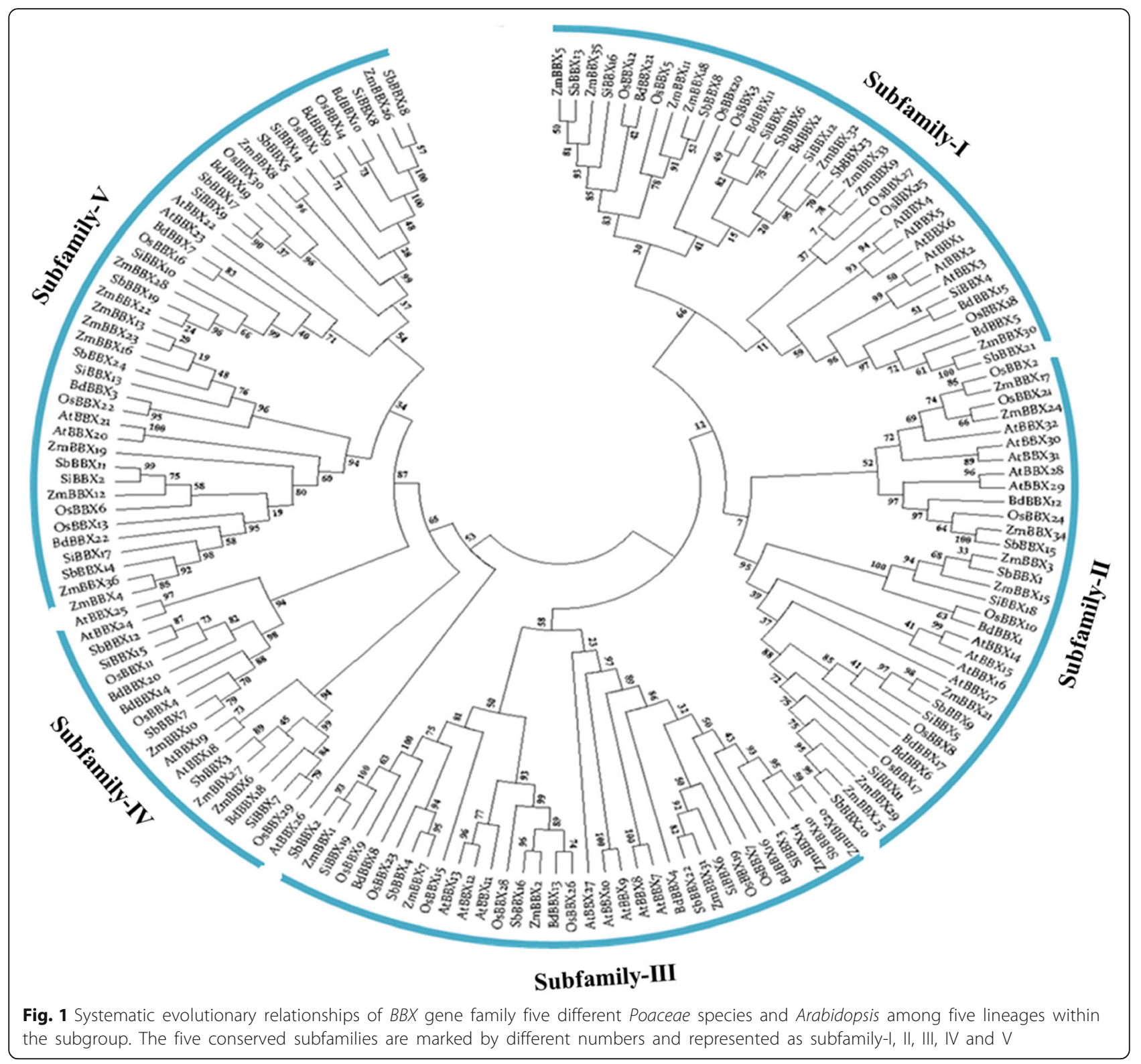

on the chromosome is varied in millet genome. However, a high number of $\operatorname{SiBBX}$ (6) genes were observed on chromosome 1, whereas the lowest number of $\operatorname{SiBBX}$ genes (1) was found on chromosome 1. 4 and 3 SiBBX members are located on chromosome 4 and 7 , respectively. $2 \mathrm{SiBBX}$ genes were investigated on each chromosome 2 and 3 (Additional file 1: Figure S3E).

Multiple online databases including Pfam, SMART, Inter Pro Scan, Conserved Domain Database (CDD), NCBI, and Scan Prosite were used to identify the conserved domains of the Poaceae BBX proteins. The family-specific domains of BBX proteins including B-box1, B-box2, and CCT conserved domains, were aligned by DNAMAN software, and their logos were constructed via Web Logo online tool (Additional file 1: Figure S4). Previous studies investigated that the CCT domains comprised are the most conserved family specific domain among B-box1, B-box2 and CCT domains (Additional file 1: Figure S5a,b,c) [4, 28], and similar results were obtained for Poaceae BBX proteins. Previously, it was also postulated that B-box1 domain is the highly conserved domain than B-box2 domain and deletion event occur in the B-box2 domain. We also found that B-box1 was more conserved compared with B-box2 domain signifying that the deletion process could happen in B-box2 domains during evolution (Additional file 1: Figure S5a, b).

The duplication of individual genes, chromosomal segment, or of the entire genome itself are the major forces during the course of genome evolution in plants [29]. We identified the possibility of gene duplication in the $B B X$ gene family in maize, rice, sorghum, stiff brome 
and millet (Fig. 2). A diagram constructed with the Circos program was used to draw the duplicated blocks in these plants genome. Both the segmental and tandem duplications were studied in this investigation. 25 $Z m B B X$ pairs were located in the segmentally duplicated regions on different chromosomes in the maize genome.

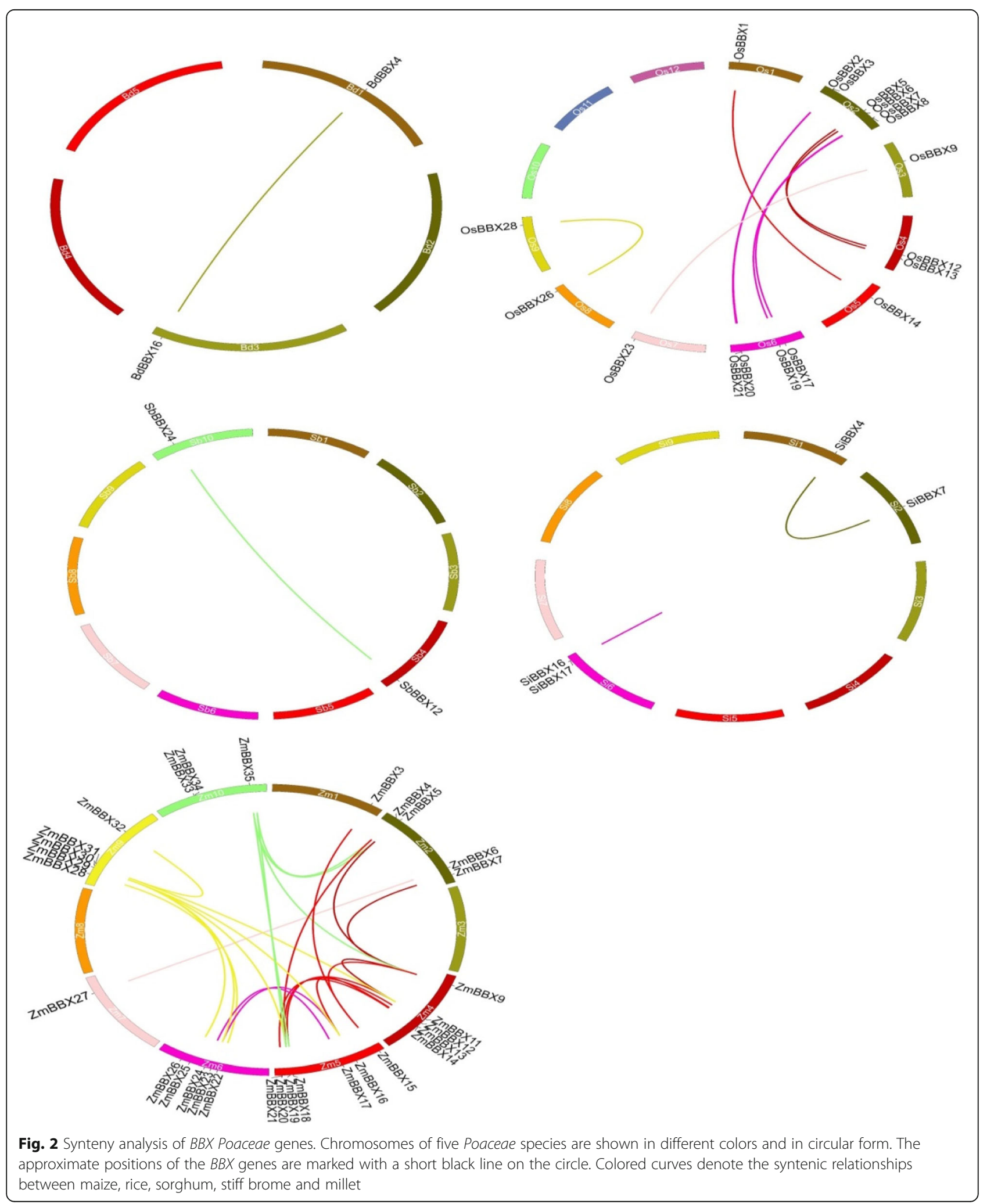


$9 O s B B X$ pairs of the duplicated region were found in the rice genome. Only one pair of the segmentally duplicated region was identified in each sorghum and stiff brome genome, whereas two pairs of the duplicated region of $B B X$ genes were located on the chromosome in millet genome. However, no tandem duplication was observed among the $B B X$ family members in the studied plants. The results indicated that only segmental duplication may take part in the evolution of $B B X$ genes in maize, rice, sorghum, stiff brome, and millet.

\section{Developmental and Tissue-Specific Expression Profiles of Rice $B B X$ Genes}

We examined the different developmental stages/tissues to study the biological roles of $B B X$ genes in the plant growth and development, based on a set of microarray data obtained from Genevestigator v3 and quantitative real-time polymerase chain reaction (qRT-PCR). The expression data from the microarray analysis of rice $B B X s$ are presented in the form of a heat map, from blue to pink reflecting the percentage expression (Fig. 3). Nine tissues including seedling, shoot, leaves, seed, endosperm, embryo, anther, pistil, pre and post-emergence inflorescences, were analyzed. The 30 candidates of rice $B B X$ genes displayed quite a similar expression profile among the tested tissues (Fig. 3). Eight members of rice $B B X$ (OsBBX4, OsBBX5, OsBBX9, OsBBX10, OsBBX11, $O s B B X 12$, OsBBX20, and OsBBX29) were highly expressed in seedling, shoot, leaves, seed-5 DAP, pistil, anther, pre and post-emergence inflorescences. No expression was detected for all the members of $B B X$ genes in endosperm and seed-10 DAP except for OsBBX7, $O s B B X 16$ and $O s B B X 29$; however, we found $17 B B X$ genes members (OsBBX1, OsBBX2, OsBBX3, OsBBX4, OsBBX5, OsBBX7, OsBBX9, OsBBX10, OsBBX11, OsBBX12, OsBBX14, OsBBX16, OsBBX19, OsBBX20, $O s B B X 22$, OsBBX24 and $O s B B X 29$ ) with high transcripts in seed-5 DAP. No or extremely low transcript level was detected for OsBBX6, OsBBX18, OsBBX28, and $O s B B X 30$ among all the studied tissues. Moreover, we observed the expression profile of two $B B X$ genes, namely $O s B B X 16$ and $O s B B X 29$, among all the tissues apart from endosperm-25 DAP, seed-10 DAP and endosperm-25 DAP (replicate). This investigation found that all the $B B X$ genes were expressed in the shoot except OsBBX15, $O s B B X 18$, OsBBX21, OsBBX23, and OsBBX28.

Furthermore, we performed qRT-PCR of the 12 rice $B B X$ members (OsBBX1, OsBBX2, OsBBX7, OsBBX8, OsBBX9, OsBBX12, OsBBX14, OsBBX16, OsBBX17, $O s B B X 19, O s B B X 21$ and $O s B B X 24)$ to find out the expression profiles among 14 different tissues (Fig. 4). The tissues were collected at three different stages: 1) seedling stage including leaf, stem and root; 2 ) booting stage consisted node-1, node-2, internode-1, internode-2, leaf sheath-1, and leaf-sheath-2; 3) heading stage including flag leaf, leaf blade, flower stage-1, flower stage- 2 and flower stage-3. The transcript levels of all the studied $B B X$ genes were high in the stem, internode- 1 , and flower stage- 3 tissues. All the $12 B B X$ members showed low transcription in the root, flag leaf, and internode- 2 tissues. No high expression was detected for the all the $B B X$ genes in node-2 except for OsBBX14, OsBBX16, $O s B B X 21$, and $O s B B X 24$. Low transcript level was observed for $O s B B X 17$ gene in leaf, whereas high transcript level was detected for the remaining $B B X$ members. The expression profile of all the $B B X$ genes was almost similar in node- 2 and internode-2. High expression profile was found for OsBBX1, OsBBX2, OsBBX7, OsBBX8, $O s B B X 12$ and $O s B B X 17$ in leaf sheath-1 and leaf sheath-2, while the rest of $B B X$ members showed low expression profile in these two tissues. In leaf blade and flowering stage-1, the expression profile of all $O s B B X$ genes was maximum except $O s B B X 8$, OsBBX12, and $O s B B X 17$. The transcription rate of all $B B X$ members was high in flowering stage-2 excluding OsBBX14, $O s B B X 17$, and OsBBX19. Overall, we noted that the transcript level of most rice $B B X$ genes was high in the heading stage, followed by booting and seedling stage based on the three stages. The present study found the expression profile (low or high) of OsBBXs in almost all the tested tissues. These findings indicated the multiple roles of $B B X$ gene family in the development and growth of rice.

\section{Inducible Expression Analysis of Rice $B B X$ Genes under Abiotic stresses and hormonal applications}

Gene expression analysis can provide essential clues for gene function; therefore, we carried out qRT-PCR to investigate the transcript levels of the rice $B B X$ genes under different abiotic stresses, including drought, cold and salt. Describing the expression profiles of all rice $B B X$ genes was exhaustively difficult; therefore, twelve $B B X$ members (OsBBX1, OsBBX2, OsBBX7, OsBBX8, OsBBX9, OsBBX12, $O s B B X 14$, OsBBX16, OsBBX17, OsBBX19, OsBBX21, and $O s B B X 24$ ) of rice $B B X$ gene family were assessed (Fig. 5). More than two-fold difference in transcript levels was considered to be the true difference for the genes under treatments. We found that the transcript levels of $O s B B X 7$, OsBBX8, OsBBX9, OsBBX12, OsBBX16, and $O s B B X 21$ were down-regulated, whereas the remaining six $B B X$ members were up-regulated at least at one (OsBBX14, OsBBX17, and OsBBX19) or two-time points (OsBBX1, OsBBX2, and OsBBX24) under drought stress. Under cold stress, the expression profile of only one $B B X$ gene (OsBBX12) was high at all the tested time points compared to $0 \mathrm{hr}$ sample (control), whereas the expression profile of OsBBX14 and OsBBX21 was down-regulated. The expression of $O s B B X 1$ and $O s B B X 2$ 


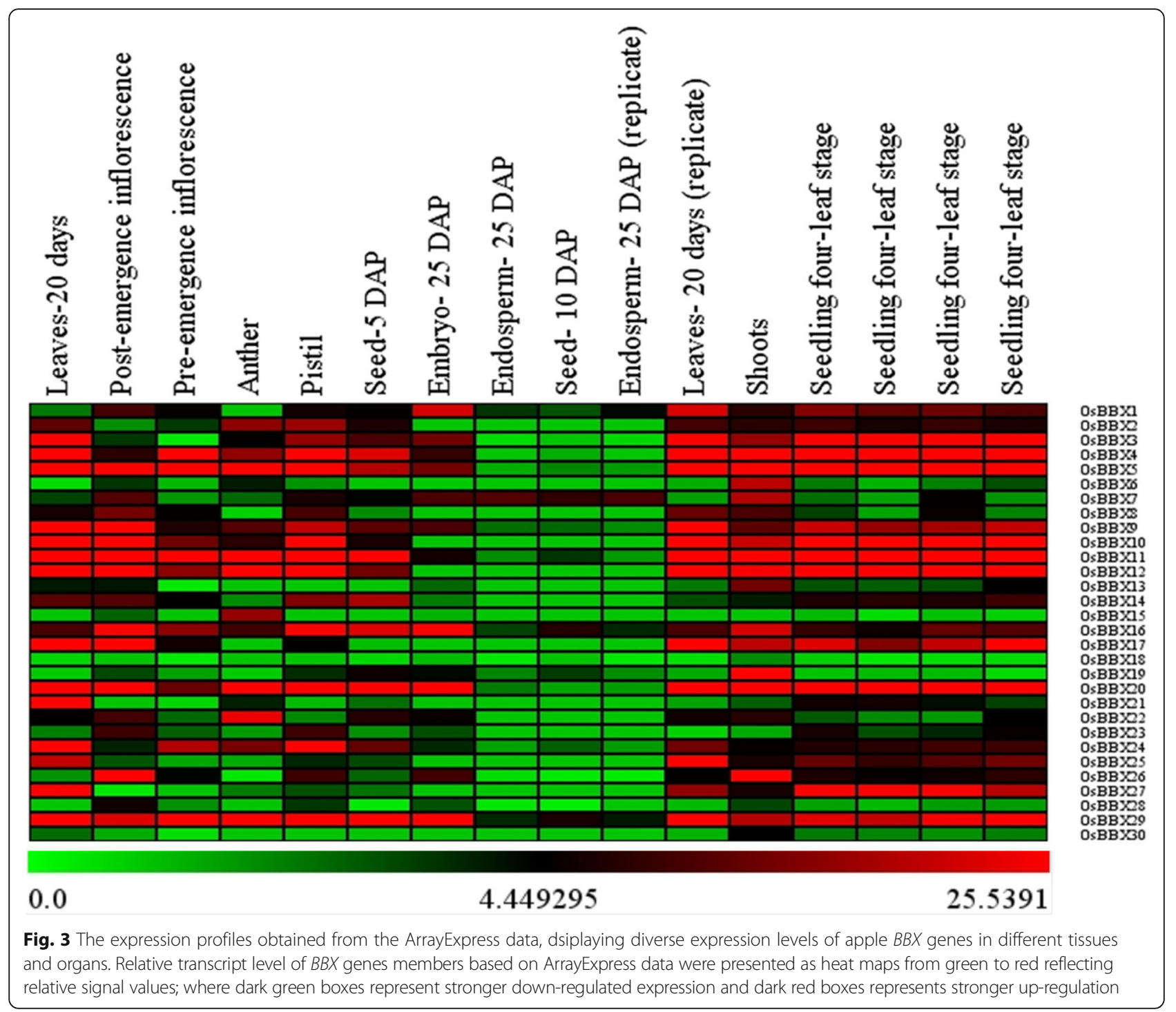

and $O s B B X 19$ was high at $3 \mathrm{hr}$ and $6 \mathrm{hr}$ time points, respectively, while the other six $B B X$ members were up-regulated at two or three time points under cold stress. Similarly, the transcript profile of $O s B B X 1, O s B B X 7$, $O s B B X 8$, and $O s B B X 16$ was high at all the time points under salt stress. Moreover, some $B B X$ members (OsBBX12, OsBBX14, OsBBX17, and OsBBX24) were down-regulated, while the rest of the four $B B X$ genes up and down-regulated at different time points under salt stress. Altogether, we observed that transcript of most rice $B B X$ members was significantly affected under salt and cold stresses; in addition, we also noticed that the $B B X$ members were also up and down-regulated at some time points under drought conditions. All these results indicate the involvement of $B B X$ gene family in plant growth and development and their response against multivariate stresses.
qRT-PCR was also used to analyze the transcript patterns of all $B B X$ genes under GA, ABA, SA, and MeJA hormones applications, to reveal the effects of various hormones on the expression of $B B X$ gene family members in rice (Fig. 6). We noticed that the expression levels of $O s B B X 1$, OsBBX17, OsBBX19, and OsBBX24 were promoted in response to exogenous GA treatment at all the time points, whereas the transcripts of $O s B B X 9$ and $O s B B X 21$ were down-regulated. Furthermore, $O s B B X 2$, $O s B B X 7$, and $O s B B X 8$ were up-regulated at 3,6 and 24 hr. We also found low transcripts for some $B B X$ members including $O s B B X 12$, OsBBX14 and $O s B B X 16$ genes under GA treatment. In contrast, the expression levels of all rice $B B X$ gene members were very low excluding OsBBX14 under ABA treatment. Moreover, the transcript levels of $O s B B X 12$, OsBBX17, and $O s B B X 19$ were up-regulated at all the time points under SA hormone, whereas OsBBX21 


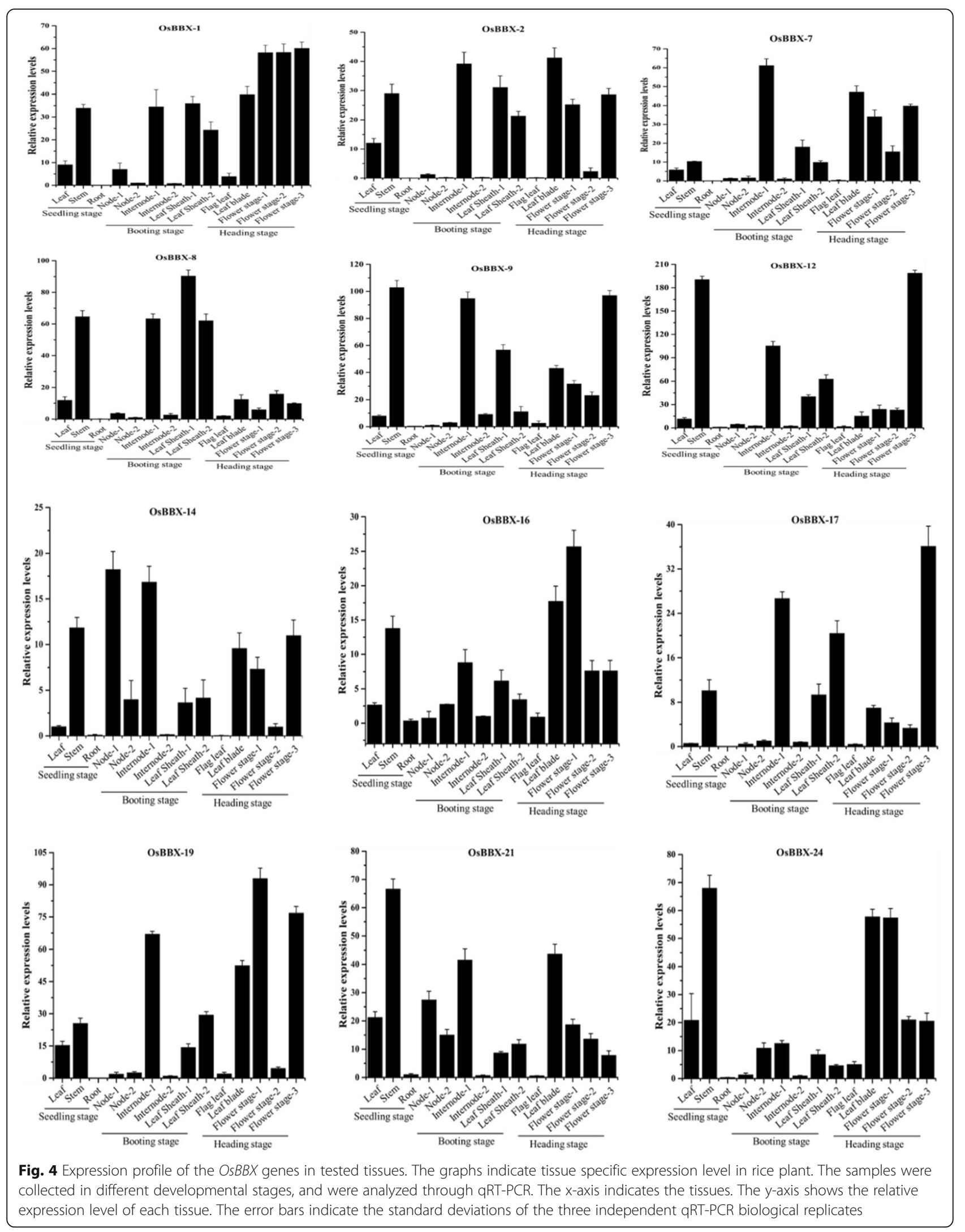




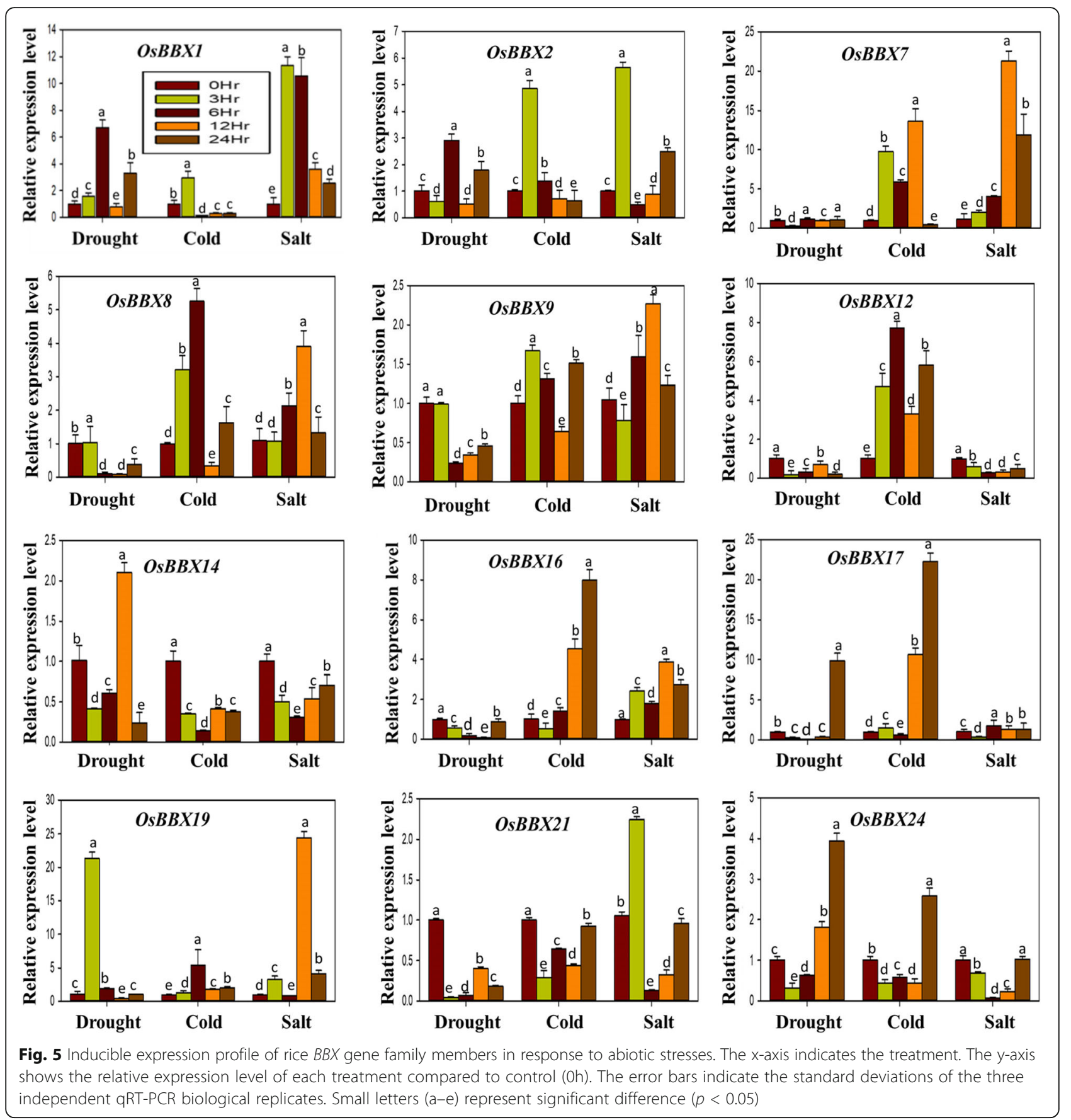

was down-regulated. We found some genes members, namely $O s B B X 2$ and $O s B B X 9$, with high expression profiles till $12 \mathrm{hr}$ post-treatment and their expression was suddenly declined at the $24 \mathrm{hr}$ time point. The expression of $O s B B X 1$ was increased at only one time point (12 hr). We also observed a maximum number of $B B X$ members shown up-regulation in expression at 3, 6 and $12 \mathrm{hr}$ time points under SA treatment. Under MeJA hormones, most rice $B B X$ was up-regulated at least one or two time points, however, $O s B B X 2$ and $O s B B X 12$ were up-regulated at all the time points. Low transcript level was detected for $O s B B X 1$ and $O s B B X 8$ at all the time points in response to exogenous MeJA treatment. Overall, the expressions of rice $B B X$ genes members were highly affected by exogenous GA, SA and MeJA hormones. Additionally, the transcripts of rice $B B X$ members were also changed by exogenous ABA treatment at a few time points. Thus, the results reveal that in response to signaling molecules the 

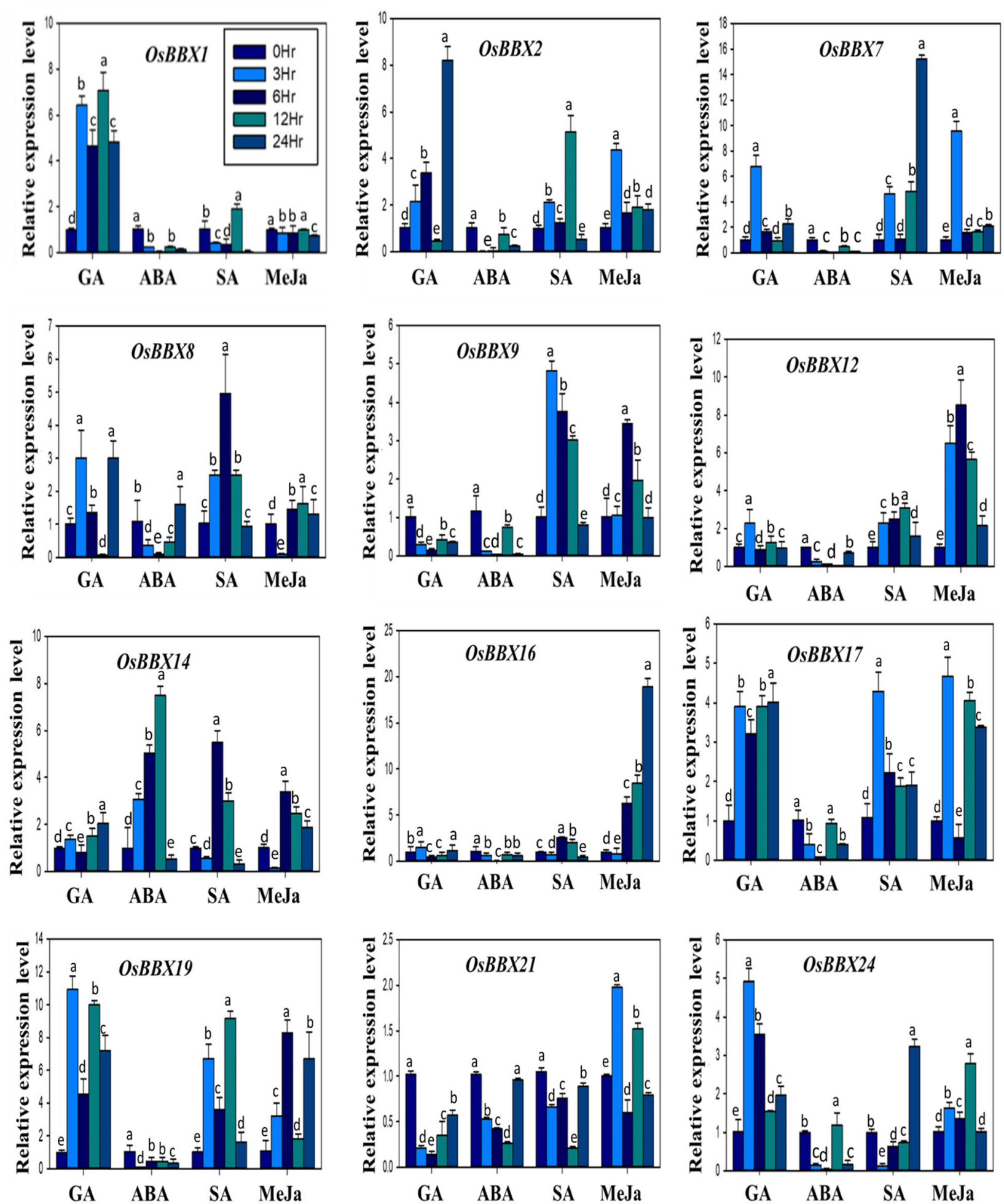

Fig. 6 Inducible expression profile of rice $B B X$ gene family under exogenous hormones. The $x$-axis indicates the treatment. The $y$-axis shows the relative expression level of each treatment compared to control (Oh). The error bars indicate the standard deviations of the three independent qRT-PCR biological replicates. Small letters $(a-e)$ represent significant difference $(p<0.05)$ 
$B B X$ genes members underwent clear variations in transcript level suggesting their hormone-induced differential responses in rice.

\section{Expression Profiles of rice $B B X$ genes under metals treatments}

Two-week-old rice plants were exposed to four different metals stressors such as $\mathrm{Cr}, \mathrm{Cd}, \mathrm{Ni}$, and $\mathrm{Fe}$, to insight the transcriptional regulation and expression profiles of rice $B B X$ genes, and the possible involvement of heavy metal stresses (Fig. 7). The temporal induction of rice $B B X$ genes members at the transcriptional level at a various time point were evaluated through qRT-PCR. We found that the transcript profiles of OsBBX1, OsBBX7, $O s B B X 8, O s B B X 17$, and $O s B B X 19$ were affected by all the four metals including $\mathrm{Cr}, \mathrm{Cd}, \mathrm{Ni}$ and $\mathrm{Fe}$ metals at some time points. The expression profiles of OsBBX2 and $O s B B X 14$ genes were up-regulated under all four metal stresses apart from $\mathrm{Cr}$ and $\mathrm{Cd}$, respectively. The transcription patterns of OsBBX9 had shown obvious changes in the expression level under $\mathrm{Ni}$ stress; likewise, $O s B B X 16$ and $O s B B X 21$ were up-regulated by Fe stress while the response of these genes to other metals such as $\mathrm{Ni}, \mathrm{Cr}$, and $\mathrm{Cd}$ was very low. Similarly, the expression level of OsBBX24 gene was high at 3 and $6 \mathrm{hr}$ under $\mathrm{Ni}$ metal, while low transcript was noticed under other three metal treatments. For OsBBX12, low transcript level was observed under $\mathrm{Ni}$ and $\mathrm{Cr}$ metal, however, the expression was up-regulated under $\mathrm{Fe}$ and $\mathrm{Cd}$ metal stresses. Based on time points, we noticed that most rice $B B X$ members were up-regulated at $12 \mathrm{hr}$ time point followed by 6,3 and $24 \mathrm{hr}$, respectively. Furthermore, based on metals, this study observed the expression of almost all the $B B X$ members shown up-regulation at least at one time point under $\mathrm{Fe}$ and $\mathrm{Ni}$ metals excluding $O s B B X 9$ and $O s B B X 21$ genes, respectively. In response to $\mathrm{Cr}$ and $\mathrm{Cd}$, rice $B B X$ genes showed a low level of expression apart from $O s B B X 8, O s B B X 12$, and $O s B B X 19$ and $O s B B X 7$ and $O s B B X 14$, respectively. Overall, the studied $B B X$ members showed high expression profiles in $\mathrm{Fe}$ and $\mathrm{Ni}$ compared with $\mathrm{Cr}$ and $\mathrm{Cd}$ metals. The unique inducible expression patterns of the $B B X$ gene family members under metal stresses may indicate the role of $B B X$ genes family in response to heavy metals. However, further studies are required to investigate deeply the particular behavior role of $B B X$ gene family in plant multivariate stresses.

\section{Discussion}

The gene clustering and evolutionary relationship mostly change due to domain shuffling and low sequence identity among the homologs proteins. Therefore, the rearrangement of domain composition, exon shuffling and gene duplication may lead to the expansion of gene families in plants during evolutionary processes $[29,30]$. Subsequently, the duplicated gene may promote functional variations, and possibly expand the functional characteristics of genes [31, 32]. Furthermore, single gene duplication might be the main cause leading to the expansion of gene families in plants.

\section{Identification and Evolution history of $B B X$ family members in various plants}

$B B X$ gene family has been got more attention from the scientific community in the past couple of years. The genome-wide identification analysis of $B B X$ genes has been already investigated in rice [14] and other important plants such as Arabidopsis, tomato, potato, pear and apple $[1,14,33-36]$. In this study, we also reported the genome-wide identification of $B B X$ genes in five Poaceae species (maize, rice, sorghum, millet, and stiff brome), and their expression analysis under abiotic (cold, drought and salt), hormones (GA, ABA, SA and MeJA) and metal stresses $(\mathrm{Cr}, \mathrm{Cd}, \mathrm{Ni}$ and $\mathrm{Fe})$ in rice. Based on our results, we found a total of $131 B B X$ genes in the five Poaceae species genomes including 36 from maize, 30 from rice, 24 from sorghum, 22 from stiff brome and 19 from millet (Table 1). The previous study also found a similar number of $B B X$ genes in the rice genome [14]. The number of $B B X$ gene family members is pretty consistent among different crop plants, such as 30, 32, 29 and $30 B B X$ genes members were already identified in rice, Arabidopsis, tomato, and potato, respectively $[1,14$, $33,36]$. The difference in the number of $B B X$ genes among the crops plants is very less. However, a total of $67 B B X$ genes in apple [35]. The difference in the number of $B B X$ genes between tree and crop plants may due to the large and heterozygous genome of apple. Furthermore, we also found less number of $B B X$ members in two species of Poaceae family, 22 from stiff brome and 19 from millet. The difference may due to the genome of these two species are not fully sequenced or may small and simple genome.

Previous studies identified 4 different types of $B B X$ proteins based on domain organization in tomato and Arabidopsis [1,36]. We also found 4 different types of BBXs (Table 2), BBXs with only one B-box domain, BBXs with two B-boxes domains, BBXs with one B-box and additional CCT domains and BBXs with two B-boxes and additional CCT domains. However, we detected a small difference in the composition of a different class of BBXs in different species. The numbers of $B B X$ with only one B-box domain, two tandem B-boxes, BOX1 plus CCT, two tandem B-boxes plus the CCT domain were 7, 8, 4, and 13, and 6, 10, 5, and 8 in Arabidopsis and tomato, respectively, however this arrangement was $3,10,10$ and 7 in rice, 4, 17, 10 and 5 in maize, 2, 8, 9 and 5 in sorghum, 1, 10, 7 and 3 in stiff 

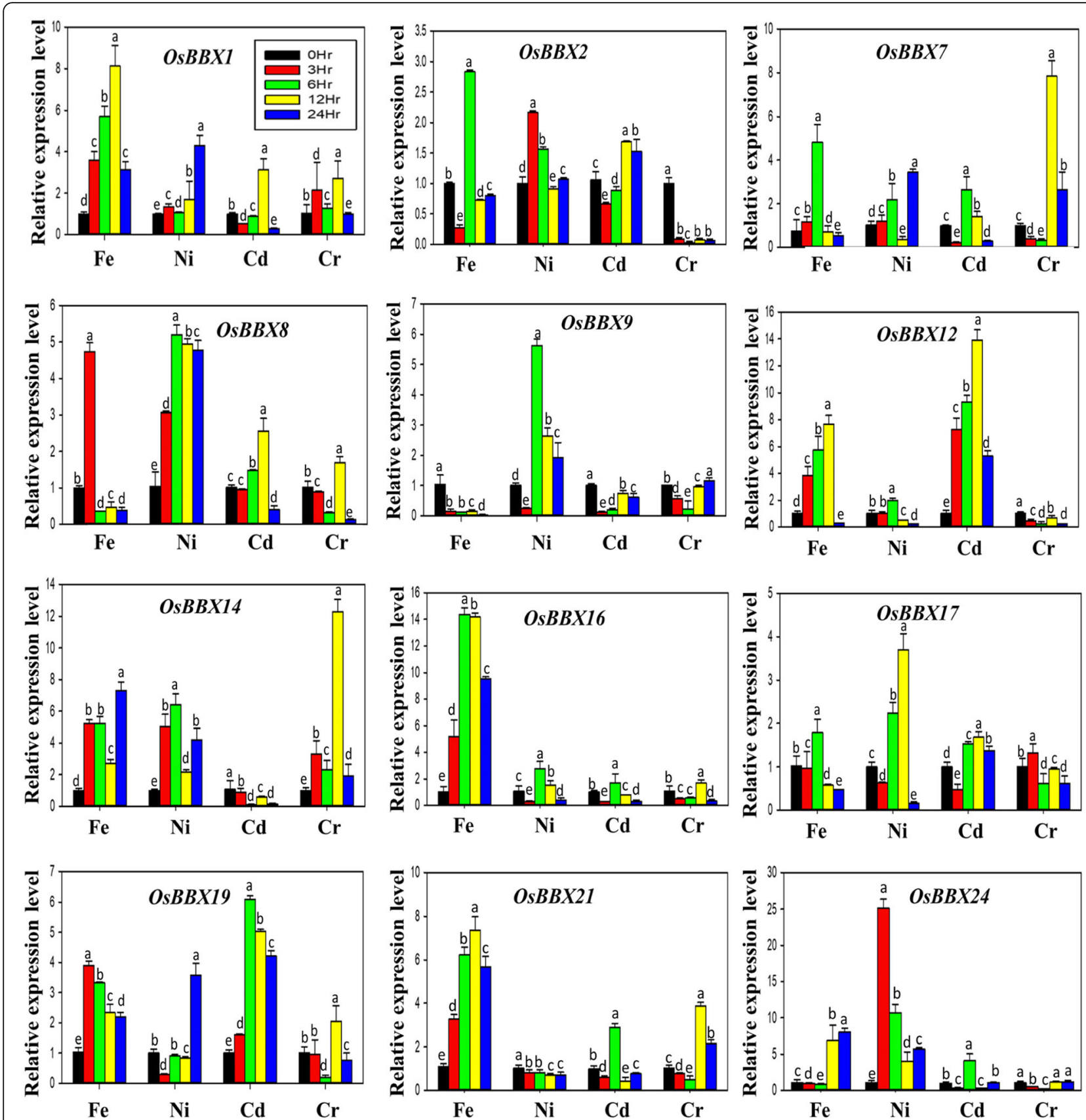

Fig. 7 Inducible expression profile of rice BBX gene family members in response to heavy metals. (A), Chromium (Cr); (B), Cadmium (Cd); (C), Nickel (Ni); (D), Iron (Fe), respectively. The $x$-axis indicates the treatment. The $y$-axis shows the relative expression level of each treatment compared to control (Oh). The error bars indicate the standard deviations of the three independent qRT-PCR biological replicates. Small letters (a-e) represent significant difference $(p<0.05)$

brome, and 1, 8, 5 and 5 in millet. The results indicate that $B B X$ gene family may share conserved gene architecture and domain organization in plants during the evolution process.

The Arabidopsis $B B X$ was clearly divided into five clusters on the basis of different conserved domains combinations [1]. Two B-boxes plus additional CCT domains containing $B B X(A t B B X 1-A t B B X 13)$ were found in group-1 and 2; one B-box plus CCT domain containing genes (AtBBX14-17) were clustered into group-3, $B B X$ genes containing two B-boxes $(A t B B X 18-25)$ and one B-box domains (AtBBX26-32) were observed in clade- 4 and 5 in Arabidopsis, respectively [1]. Whereas, in five Poaceae species, maximum number of one and two B-boxes and additional CCT conserved domains containing $B B X$ genes members were cluster together 
into subfamily I, II and III (Fig. 1), $B B X$ genes possessing one B-box domain were detected in subfamily II, IV and V, whereas two B-boxes containing $B B X$ genes were observed in subfamily IV and $V$ in this study. The classification of Poaceae BBX members based on conserved domain was relatively difficult. The reason behind uneven distribution may due to a large number of genes or the small difference in the domain organization in the plant species. For instance, we noticed that $7 B B X$ genes possessed only one B-box domain, $8 B B X$ members had two B-boxes domain, 4 $B B X$ members contained one B-box and additional CCT domain and $13 B B X$ genes were found with two B-boxes and additional CCT domains in Arabidopsis [1]. In contrary, $3 B B X$ possessed only one B-box domain, $10 B B X$ found having two B-boxes domains, one B-box and additional CCT domain were observed in $10 B B X$ members and $7 B B X$ genes comprised of two B-boxes and additional CCT domains in rice (Table 2). Similar differences were also observed for B-box genes in other four studied Poaceae species. However, we also noted that the gene structure and functional characteristic of $B B X$ genes within the subfamily was quite similar. Thus, it is assumed that $B B X$ members share a similar gene structure and functional characteristic within the same subfamily during the evolutionary relationship. Previously, it also has been reported that FRO gene family members in rice shared similar gene structure and functional characteristic during evolution in rice [37].

Moreover, It has been already reported that CCT is the highly conserved domain $[29,38]$. The alignment of B-box1, B-box2 and CCT domain also indicated that the CCT domain was highly conserved compared with B-box1 and B-box2 domain (Additional file 1: Figure S5a, b, c). However, a theory has been proposed that a deletion process occurs during the evaluation that leads to making another class of $B B X$ genes, containing only one B-box domain [3]. After detail sequence alignment of two B-box domains (B-box1 and B-box2) revealed that B-box1 domain was highly conserved compared with B-box2 in rice $B B X$ (Additional file 1: Figure S5a, b), thus, it's postulated that deletion process could occur in the B-box2 domain and give birth to the B-box1 domain.

Large-scale duplication and tandem duplication processes are vital for the amplification of gene family members in the genome during the evolution [39]. In this study, both the tandem and segmental duplication events were analyzed to study the evaluation of the $B B X$ genes in Poaceae. We found only segmental duplication in the $B B X$ genes (Fig. 2) indicating that segmental duplication events took part in the expansion of the $B B X$ gene family in Poaceae.
Tissue-Specific gene expression profiles reveal the diverse roles of $B B X$ gene family in plant growth and development

The specific gene family members have common genes expression profile features in plants. This may coordinate and/or differ in the functional interaction of the family members. It was previously reported that BBX proteins control the diverse functions of the plant, such as photomorphogenesis, flowering and shade avoidance [40, 41]. In Arabidopsis, the overexpression of a $B B X$ gene (BBX6, COL5) promotes early flowering [42], and the overexpression COL9 (BBX7) delay the flowering under SD (short day) condition [43]. $B B X$ homologous genes which contribute to different biological processes with obvious tissue specificity in gene expression have been functionally characterized in maize [44]. The members of $B B X$ gene family also showed diverse expression in all the tested tissues in tomato [36]. Similarly, in potato maximum number of $B B X$ family members was detected with distinct expression pattern among the tested organs [33]. Likewise, we investigated the expression of $B B X$ family in 14 different tissues and the samples were collected at three different stages, seedling stage root, booting stage and heading stage (Fig. 4). We found that the expression of almost all the $B B X$ members was high in all the tested samples apart from roots. Furthermore, we also noticed that the transcript levels of the studied $B B X$ members were high in the heading stage. Moreover, the database searching found that $B B X$ gene more expressed seedling, leaf, shoot and flowering-related tissues (Fig. 3). Thus, the database searching and functional prediction of $B B X$ gene family members in various tissues and different developmental stages demonstrate that $B B X$ gene family might play vital roles in plant growth, and some $B B X$ genes members might have a unique function in specific developmental stages.

\section{Pronounced but differentiated inducible expression patterns under a number of environmental, hormonal and metal stresses imply the vital contributions of $B B X$ gene members to multivariate stress tolerance}

Various adverse environmental aspects such as ion toxicity, salinity, drought, extreme temperatures negatively disturb plant growth and development [45-47]. Among them, several abiotic stresses cause general or specific effects on growth and development and changes at the transcriptional level in plants [48-50]. Here, we detected that rice $B B X$ genes are sensitive to a set of abiotic stresses, and their transcriptional expressions were greatly altered by salt, cold, drought, GA, SA, MeJA, $\mathrm{ABA}$ and metals stress treatments, displaying their contribution in responses to multiple stresses in rice. Several investigations have proposed that $B B X$ genes are important for the photoperiodic regulation of flowering, 
seedling photomorphogenesis, shade avoidance, and responses to biotic and abiotic stresses. It has been also stated that the salt tolerance protein STO (AtBBX24) enhances the growth of root under a high salinity condition in Arabidopsis [15] and the salt tolerant activities was also triggered in yeast cells [16]. AtBBX18 acts as negative regulator both in photomorphogenesis and thermotolerance in Arabidopsis [12]. Furthermore, $B B X 18$ negatively regulates the expression of heat-responsive genes such as DGD1, Hsp70, Hsp101, and $A P X 2$, thereby reducing germination and seedling survival after a heat treatment [12]. In Chrysanthemum, CmBBX24 performs a dual function, delaying flowering and also increasing cold or drought tolerance in the plant [19]. Moreover, some studied found that BBX proteins also involve in hormones signaling. A recent investigation found that the expression pattern of $B B X$ genes was altered in response to $\mathrm{ABA}$ and cyclic ADP-ribose (cADPR) temperatures [6, 7]. The involvement of $B B X$ genes in the COP/HY5 signaling pathway indicates that $B B X 18$ may work as an integrator of both GA and COP1/HY5 pathways [13]. Based on the previous studies, we evaluated the expression of $O s B B X$ genes in response to numerous abiotic and hormonal stresses and found that the most rice $B B X$ members show high expression levels under abiotic stresses (Fig. 5). The expression patterns of $O s B B X 1, O s B B X 2$, and $O s B B X 19$ genes were affected by all the three used abiotic stresses including drought, salt and cold stresses. OsBBX7, $O s B B X 8$, and $O s B B X 16$ genes showed high expression under salt and cold conditions, whereas OsBBX17 and $O s B B X 24$ genes were up-regulated in response to drought and cold. In addition, we found that most rice $B B X$ genes were up-regulated under the cold and salt condition, while, less transcript level was observed for most rice $B B X$ genes in response to drought. The members of rice $B B X$ gene family also showed maximum expression levels in response to different hormones (Fig. 6). The expression of $O s B B X 2$, OsBBX7, OsBBX17, $O s B B X 19$, and $O s B B X 24$ genes were strongly triggered in response to GA, SA and MeJa hormones. Similarly, $O s B B X 1$ and $O s B B X 16$ genes displayed high expression under GA and MeJa hormones, respectively. Moreover, the transcript levels of $O s B B X 8$ and $O s B B X 14$ were promoted under GA, ABA, SA and MeJa hormones. Although most rice $B B X$ genes were up-regulated at different points under GA, SA and MeJA hormones, the transcripts of the $B B X$ gene family were less effected by ABA. Furthermore, the transcript levels of most $B B X$ members were significantly stimulated by heavy metal stresses even though somewhat unique responses occurred for some members under certain metals (Fig. 7). For example, the transcript profiles of OsBBX1, OsBBX7, $O s B B X 8, O s B B X 17$, and $O s B B X 19$ members were greatly affected by $\mathrm{Fe}, \mathrm{Ni}, \mathrm{Cr}$, and $\mathrm{Cd}$ metals, however, the transcription activity of OsBBX24 was significantly changed in response to all the applied metals apart from $\mathrm{Cr}$ metal. Similarly, the transcript profile of OsBBX14 was enhanced in response to all used metals except $\mathrm{Cd}$ metal. Furthermore, we also found some $B B X$ genes which showed high expression profile in response to only one metal, for instance, OsBBX9 was highly expressed under $\mathrm{Ni}$ metal. Overall, the results obtained here suggest that $B B X$ gene family may perform several functions in plant growth and development and in response to abiotic, metal stresses and hormonal applications although their exact role remains unclear. Further experiments need to be done to investigate the exact role of $B B X$ gene family in plant growth and development.

\section{Conclusions}

Over a long evolutionary relationship of plants, $B B X$ genes had shown consistency in their common characteristics and functional behavior. In this context, the differential expression patterns of $B B X$ genes in Poaceae plants play a vital role in the plant growth regulation. The regulatory mechanism and transcriptional variation of $B B X$ genes are highly responsive to external factors, thus, the multivariate stresses and hormonal application substantially triggered the up-regulation of the differentially expressed genes, thereby participating the beneficial allocation and potential role of these genes in plants. We suggest that the specific role of particular $B B X$ gene should be a target for defining the stress response, functional divergence and possible crosstalk in plants such as rice.

\section{Additional file}

Additional file 1: Supplementary Figures and Supplementary tables. (DOCX $5100 \mathrm{~kb}$ )

\section{Abbreviations}

GA: Gibberellic acid; ABA: abscisic acid; MeJA: methyl jasmonate; SA: salicylic acid; Ni: Nickle; Fe: Iron; Cd: Cadmium; Cr: Chromium

Acknowledgements

Not applicable

\section{Funding}

This work was supported by the National Natural Science Foundation of China (grant no. 31770204 and 31270299) and the Program for New Century Excellent Talents in University of China (NCET-11-0440).

\section{Availability of data and materials}

The datasets generated during the current study are available within the article and additional files.

\section{Authors' contributions}

AS, X.-QJ and K-MC designed the research; AS and X-QJ conducted the experimental work, SY, M-RZ, X-YW and Q-QC contributed to the preparation of biological materials, I.M. performed bioinformatics analysis, and AS, W-QL, W-TL and K-MC wrote the manuscript. All authors read and approved the final manuscript. 


\section{Ethics approval and consent to participate}

All the available materials used in our study were grown in the field of State Key Laboratory of Crops Stress Biology for Arid Areas (Northwest A\&F University, Yangling, China). Samples collection complied with the institutional, national and international guidelines. This article did not contain any studies with human participants or animals performed by any of authors. No specific permits were required.

\section{Consent for publication}

Not applicable.

\section{Competing interests}

The authors declare that they have no competing interests.

\section{Publisher's Note}

Springer Nature remains neutral with regard to jurisdictional claims in published maps and institutional affiliations.

\section{Received: 27 August 2018 Accepted: 29 November 2018}

\section{Published online: 09 January 2019}

\section{References}

1. Khanna R, Kronmiller B, Maszle DR, Coupland G, Holm M, Mizuno T, Wu S-H. The Arabidopsis B-box zinc finger family. Plant Cell. 2009;21 (11):3416-20.

2. Massiah MA, Matts JA, Short KM, Simmons BN, Singireddy S, Yi Z, Cox TC. Solution structure of the MID1 B-box2 CHC (D/C) C2H2 zinc-binding domain: insights into an evolutionarily conserved RING fold. J Mol Biol. 2007;369(1):1-10.

3. Crocco CD, Botto JF. BBX proteins in green plants: insights into their evolution, structure, feature and functional diversification. Gene. 2013;531(1): 44-52.

4. Gendron JM, Pruneda-Paz JL, Doherty CJ, Gross AM, Kang SE, Kay SA. Arabidopsis circadian clock protein, TOC1, is a DNA-binding transcription factor. Proc Natl Acad Sci. 2012;109(8):3167-72.

5. Cutler SR, Rodriguez PL, Finkelstein RR, Abrams SR. Abscisic acid: emergence of a core signaling network. Annu Rev Plant Biol. 2010;61:651-79.

6. Sánchez JP, Duque P, Chua NH. ABA activates ADPR cyclase and CADPR induces a subset of ABA-responsive genes in Arabidopsis. Plant J. 2004; 38(3):381-95.

7. Soitamo AJ, Piippo M, Allahverdiyeva Y, Battchikova N, Aro E-M. Light has a specific role in modulating Arabidopsis gene expression at low temperature. BMC Plant Biol. 2008;8(1):13.

8. Taki N, Sasaki-Sekimoto Y, Obayashi T, Kikuta A, Kobayashi K, Ainai T, Yagi K, Sakurai N, Suzuki H, Masuda T. 12-oxo-phytodienoic acid triggers expression of a distinct set of genes and plays a role in wound-induced gene expression in Arabidopsis. Plant Physiol. 2005;139(3):1268-83.

9. Sun Y, Fan X-Y, Cao D-M, Tang W, He K, Zhu J-Y, He J-X, Bai M-Y, Zhu S, Oh E. Integration of brassinosteroid signal transduction with the transcription network for plant growth regulation in Arabidopsis. Dev Cell. 2010;19(5): 765-77.

10. Libault M, Wan J, Czechowski T, Udvardi M, Stacey G. Identification of 118 Arabidopsis transcription factor and 30 ubiquitin-ligase genes responding to chitin, a plant-defense elicitor. Mol Plant Microbe Interact. 2007;20(8):900-11.

11. Luo X-M, Lin W-H, Zhu S, Zhu J-Y, Sun Y, Fan X-Y, Cheng M, Hao Y, Oh E, Tian M. Integration of light-and brassinosteroid-signaling pathways by a GATA transcription factor in Arabidopsis. Dev Cell. 2010;19(6):872-83.

12. Wang $Q$, Tu X, Zhang J, Chen X, Rao L. Heat stress-induced BBX18 negatively regulates the thermotolerance in Arabidopsis. Mol Biol Rep. 2013; 40(3):2679-88

13. Weller JL, Hecht V, Vander Schoor JK, Davidson SE, Ross JJ. Light regulation of gibberellin biosynthesis in pea is mediated through the COP1/HY5 pathway. Plant Cell. 2009;21(3):800-13.

14. Huang J, Zhao X, Weng X, Wang L, Xie W. The rice B-box zinc finger gene family: genomic identification, characterization, expression profiling and diurnal analysis. PLoS One. 2012;7(10):e48242.

15. Nagaoka S, Takano T. Salt tolerance-related protein STO binds to a Myb transcription factor homologue and confers salt tolerance in Arabidopsis. J Exp Bot. 2003;54(391):2231-7.

16. Lippuner V, Cyert MS, Gasser CS. Two classes of plant CDNA clones differentially complement yeast calcineurin mutants and increase salt tolerance of wild-type yeast. J Biol Chem. 1996;271(22):12859-66.
17. Belles-Boix E, Babiychuk E, Van Montagu M, Inzé D, Kushnir S. CEO1, a new protein from Arabidopsis thaliana, protects yeast against oxidative damage. FEBS Lett. 2000;482(1-2):19-24.

18. Fujibe T, Saji H, Arakawa K, Yabe N, Takeuchi Y, Yamamoto KT. A methyl viologen-resistant mutant of Arabidopsis, which is allelic to ozone-sensitive rcd1, is tolerant to supplemental ultraviolet-B irradiation. Plant Physiol. 2004; 134(1):275-85.

19. Yang Y, Ma C, Xu Y, Wei Q, Imtiaz M, Lan H, Gao S, Cheng L, Wang M, Fei Z. A zinc finger protein regulates flowering time and abiotic stress tolerance in chrysanthemum by modulating gibberellin biosynthesis. Plant Cell. 2014; 26(5):2038-54.

20. Schultz J, Milpetz F, Bork P, Ponting CP. SMART, a simple modular architecture research tool: identification of signaling domains. Proc Natl Acad Sci. 1998;95(11):5857-64.

21. Bailey TL, Williams N, Misleh C, Li WW. MEME: discovering and analyzing DNA and protein sequence motifs. Nucleic Acids Res. 2006;34(suppl_2): W369-73.

22. Hu B, Jin J, Guo A-Y, Zhang H, Luo J, Gao G. GSDS 2.0: an upgraded gene feature visualization server. Bioinformatics. 2014;31(8):1296-7.

23. Hoagland DR, Arnon DI. The water-culture method for growing plants without soil, Circular California agricultural experiment station. 2nd ed; 1950. p. 347.

24. Zhang M, Liu B. Identification of a rice metal tolerance protein OsMTP11 as a manganese transporter. PloS one. 2017;12(4):e0174987.

25. Saifullah BS, Waraich EA. Effects of lead forms and organic acids on the growth and uptake of lead in hydroponically grown wheat. Commun Soil Sci Plant Anal. 2013;44(21):3150-60.

26. Chang S, Puryear J, Cairney J. A simple and efficient method for isolating RNA from pine trees. Plant Mol Biol Report. 1993;11(2):113-6.

27. Finn RD, Bateman A, Clements J, Coggill P, Eberhardt RY, Eddy SR, Heger A, Hetherington K, Holm L, Mistry J. Pfam: the protein families database. Nucleic Acids Res. 2013;42(D1):D222-30.

28. Yan $\mathrm{H}$, Marquardt $\mathrm{K}$, Indorf $\mathrm{M}$, Jutt $\mathrm{D}$, Kircher $\mathrm{S}$, Neuhaus $\mathrm{G}$, RodríguezFranco M. Nuclear localization and interaction with COP1 are required for STO/BBX24 function during photomorphogenesis. Plant Physiol. 2011;156(4): $1772-82$.

29. Magadum S, Banerjee U, Murugan P, Gangapur D, Ravikesavan R. Gene duplication as a major force in evolution. J Genet. 2013;92(1):155-61.

30. Bedard K, Lardy B, Krause K-H. NOX family NADPH oxidases: not just in mammals. Biochimie. 2007;89(9):1107-12.

31. Morgante M, Brunner S, Pea G, Fengler K, Zuccolo A, Rafalski A. Gene duplication and exon shuffling by helitron-like transposons generate intraspecies diversity in maize. Nature Genet. 2005;37(9):997.

32. Kaessmann $\mathrm{H}$. Origins, evolution and phenotypic impact of new genes. Genome Res. 2010;20(10):1313-26.

33. Talar U, Kiełbowicz-Matuk A, Czarnecka J, Rorat T. Genome-wide survey of B-box proteins in potato (Solanum tuberosum) -Identification, characterization and expression patterns during diurnal cycle, etiolation and de-etiolation. PloS one. 2017;12(5):e0177471.

34. Cao Y, Han Y, Meng D, Li D, Jiao C, Jin Q, Lin Y, Cai Y. B-BOX genes: genome-wide identification, evolution and their contribution to pollen growth in pear (Pyrus bretschneideri Rehd.). BMC Plant Biol. 2017;17(1):156.

35. Shalmani A, Fan S, Jia P, Li G, Muhammad I, Li Y, Sharif R, Dong F, Zuo X, Li K. Genome Identification of B-BOX Gene Family Members in Seven Rosacea Species and Their Expression Analysis in Response to Flower Induction in Malus domestica. Mol. 2018;23:1763.

36. Chu Z, Wang X, Li Y, Yu H, Li J, Lu Y, Li H, Ouyang B. Genomic organization, phylogenetic and expression analysis of the B-BOX gene family in tomato. Front Plant Sci. 2016;7:1552.

37. Muhammad I, Jing X-Q, Shalmani A, Ali M, Yi S, Gan P-F, Li W-Q, Liu W-T, Chen K-M. Comparative in Silico Analysis of Ferric Reduction Oxidase (FRO) Genes Expression Patterns in Response to Abiotic Stresses, Metal and Hormone Applications. Mol. 2018;23(5):1163.

38. Yan $\mathrm{H}$, Marquardt $\mathrm{K}$, Indorf $\mathrm{M}$, Jutt $\mathrm{D}$, Kircher $\mathrm{S}$, Neuhaus $\mathrm{G}$, Rodríguez-Franco M: Nuclear localization and interaction with COP1 are required for STO/BBX24 function during photomorphogenesis. Plant physiology 2011:pp. 111.180208.

39. Cannon SB, Mitra A, Baumgarten A, Young ND, May G. The roles of segmental and tandem gene duplication in the evolution of large gene families in Arabidopsis thaliana. BMC Plant Biol. 2004;4(1):10.

40. González-Schain ND, Díaz-Mendoza M, Żurczak M, Suárez-López P. Potato CONSTANS is involved in photoperiodic tuberization in a graft-transmissible manner. Plant J. 2012;70(4):678-90. 
41. Crocco CD, Holm M, Yanovsky MJ, Botto JF. Function of B-BOX under shade. Plant Signal Behav. 2011;6(1):101-4.

42. Hassidim M, Harir Y, Yakir E, Kron I, Green RM. Over-expression of CONSTANS-LIKE 5 can induce flowering in short-day grown Arabidopsis. Planta. 2009;230(3):481-91.

43. Cheng XF, Wang ZY. Overexpression of COL9, a CONSTANS-LIKE gene, delays flowering by reducing expression of $\mathrm{CO}$ and $\mathrm{FT}$ in Arabidopsis thaliana. Plant J. 2005;43(5):758-68.

44. Li W, Wang J, Sun Q, Li W, Yu Y, Zhao M, Meng Z. Expression analysis of genes encoding double B-box zinc finger proteins in maize. Funct Integr Genomics. 2017;17(6):653-66.

45. Rengasamy P. World salinization with emphasis on Australia. J Exp Bot 2006;57(5):1017-23.

46. Cramer GR, Urano K, Delrot S, Pezzotti M, Shinozaki K. Effects of abiotic stress on plants: a systems biology perspective. BMC Plant Biol. 2011;11(1):163.

47. Stein R, Duarte G, Spohr M, Lopes S, Fett J. Distinct physiological responses of two rice cultivars subjected to iron toxicity under field conditions. Ann Appl Biol. 2009;154(2):269-77.

48. Wang G-F, Li W-Q, Li W-Y, Wu G-L, Zhou C-Y, Chen K-M. Characterization of rice NADPH oxidase genes and their expression under various environmental conditions. Int J Mol Sci. 2013;14(5):9440-58

49. Chinnusamy V, Zhu J, Zhu J-K. Cold stress regulation of gene expression in plants. Trends Plant sci. 2007;12(10):444-51.

50. Nakashima K, Ito Y, Yamaguchi-Shinozaki K. Transcriptional regulatory networks in response to abiotic stresses in Arabidopsis and grasses. Plant Physiol. 2009;149(1):88-95.

Ready to submit your research? Choose BMC and benefit from:

- fast, convenient online submission

- thorough peer review by experienced researchers in your field

- rapid publication on acceptance

- support for research data, including large and complex data types

- gold Open Access which fosters wider collaboration and increased citations

- maximum visibility for your research: over $100 \mathrm{M}$ website views per year

At $\mathrm{BMC}$, research is always in progress.

Learn more biomedcentral.com/submissions 